University of Louisville

ThinkIR: The University of Louisville's Institutional Repository

Electronic Theses and Dissertations

$5-2021$

\title{
Adaptive control and parameter-dependent anti-windup compensation for inertia varying quadcopters.
}

Benjamin Edwards Farber

University of Louisville

Follow this and additional works at: https://ir.library.louisville.edu/etd

Part of the Controls and Control Theory Commons

\section{Recommended Citation}

Farber, Benjamin Edwards, "Adaptive control and parameter-dependent anti-windup compensation for inertia varying quadcopters." (2021). Electronic Theses and Dissertations. Paper 3629.

https://doi.org/10.18297/etd/3629

This Master's Thesis is brought to you for free and open access by ThinkIR: The University of Louisville's Institutional Repository. It has been accepted for inclusion in Electronic Theses and Dissertations by an authorized administrator of ThinkIR: The University of Louisville's Institutional Repository. This title appears here courtesy of the author, who has retained all other copyrights. For more information, please contact thinkir@louisville.edu. 


\title{
ADAPTIVE CONTROL AND PARAMETER-DEPENDENT ANTI-WINDUP COMPENSATION FOR INERTIA VARYING QUADCOPTERS
}

\author{
By
}

Benjamin Edwards Farber

B.S., University of Louisville, 2019

\author{
A Thesis \\ Submitted to the Faculty of the \\ J.B. Speed School of Engineering of the University of Louisville \\ in Partial Fulfillment of the Requirements \\ for the Degree of \\ Master of Science \\ in Mechanical Engineering \\ Department of Mechanical Engineering \\ University of Louisville \\ Louisville, Kentucky
}

May 2021 



\title{
ADAPTIVE CONTROL AND PARAMETER-DEPENDENT ANTI-WINDUP COMPENSATION FOR INERTIA VARYING QUADCOPTERS
}

\author{
By \\ Benjamin Edwards Farber \\ B.S., University of Louisville, 2019
}

A Thesis Approved on

April 27, 2021

by the following Thesis Committee:

Dr. Christopher Richards, Thesis Director

Dr. Kevin Murphy

Dr. Tamer Inanc 


\section{DEDICATION}

This thesis is dedicated to

\section{Halle Reid}

who has supported and encouraged me as I worked to complete my degree.

At the most challenging points, she motivated me to keep working hard. 


\section{ACKNOWLEDGMENTS}

I would like to thank NASA Kentucky who supported this work under NASA award number NNX15AR69H. A special thanks goes to Dr. Chris Richards for giving me the opportunity to research a field of my interest. He continually provided me with guidance on all of the hardest problems that I came across while working on my thesis. I also would like to thank committee members Dr. Kevin Murphy and Dr. Tamer Inanc for teaching some of my favorite classes in my graduate studies. Finally, I would like to thank my parents Jess and Sandra Farber for all the support they have provided throughout my entire education. 


\section{ABSTRACT \\ ADAPTIVE CONTROL AND PARAMETER-DEPENDENT ANTI-WINDUP \\ COMPENSATION FOR INERTIA VARYING QUADCOPTERS}

Benjamin Edwards Farber

April 27, 2021

A novel parameter-dependent anti-windup compensator is developed to improve the performance of a saturation constrained model reference adaptive controller. The combined control structure solves the input saturation and stability problem for inertia varying quadcopters. The control synthesis follows the conventional two-step anti-windup design paradigm where a nominal controller is designed without consideration of the input saturation, and the anti-windup compensator is designed to minimize deviations from nominal performance caused by saturated inputs. To account for varying inertia of the quadcopter during package retrieval/delivery routines, the inertia parameters of the vehicle/package are estimated with an online recursive system identification technique, and these estimates are used to schedule the parameter-dependent anti-windup compensator. The performance and stability conditions of the parameter-dependent anti-windup compensator are formulated as a set of parameter-dependent linear matrix inequalities. When solved, the linear matrix inequalities yield a gain-scheduled anti-windup compensator that ensures stability and minimizes the deviation from nominal model reference adaptive control performance when saturation occurs. The effectiveness of the combined control scheme is demonstrated by simulations of an input constrained quadcopter lifting a payload of unknown mass. 
TABLE OF CONTENTS

PAGE

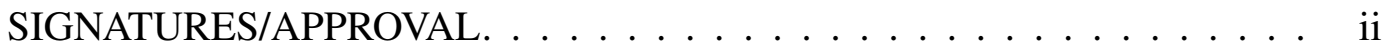

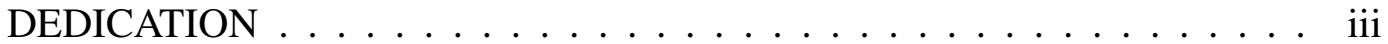

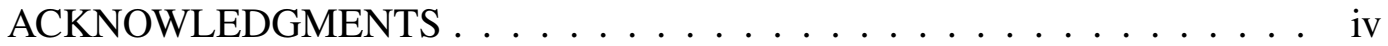

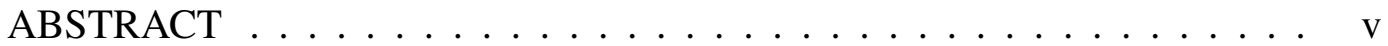

LIST OF FIGURES $\ldots \ldots \ldots \ldots \ldots \ldots \ldots$ vii

$1 \quad$ INTRODUCTION $\ldots \ldots \ldots \ldots \ldots \ldots \ldots \ldots \ldots$

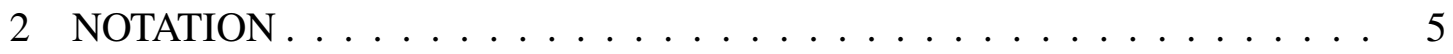

3 COMBINED MRAC AND PDAWC CONTROL SYNTHESIS . . . . . . . 6

3.1 Model Reference Adaptive Control. . . . . . . . . . . . . . . . . . 8

3.2 Parameter-Dependent Anti-Windup Compensation . . . . . . . . . . . . 14

3.2.1 Local Stability . . . . . . . . . . . . . . . . . . . . 18

3.3 System Identification. . . . . . . . . . . . . . . . . . . . . 19

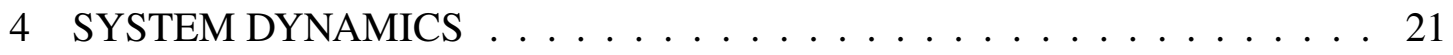

5 CONTROL SYSTEM DESIGN AND SIMULATIONS . . . . . . . . 25

5.1 MRAC Design . . . . . . . . . . . . . . . . 27

5.2 PDAWC Design . . . . . . . . . . . . . . . . . . 29

5.3 Simulation Results . . . . . . . . . . . . . . . . . . . . . 31

6 CONCLUSIONS ....................... 35

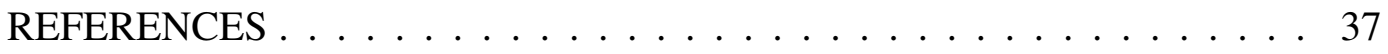

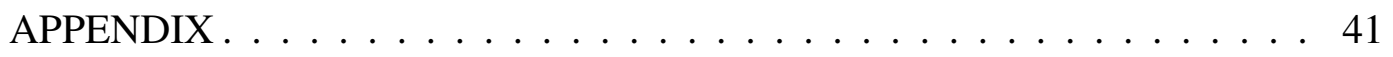

CURRICULUM VITA. . . . . . . . . . . . . . . . . . 55 


\section{LIST OF FIGURES}

FIGURE

PAGE

1 Combined MRAC and PDAWC architecture. . . . . . . . . 6

2 General MRAC system. . . . . . . . . . . . . . . . . 8

$3 \quad$ MRAC with Integral Feedback. . . . . . . . . . . . . . . . . 9

4 Equivalent representation of Fig. 1 when $\Theta(\rho)$ is parameterized by transfer functions $G(\rho)$ and $M(\rho) \ldots \ldots \ldots \ldots \ldots$

5 Quadcopter Model. . . . . . . . . . . . . . . 21

6 RLS parameter estimation. $\_1 / \hat{\rho}_{i} C_{\hat{K}} ;-1 / \hat{\rho}_{i} C_{\hat{K}, \Theta} ;-1 / \rho_{i} . \ldots . . .32$

$7 \quad$ Effect of $W_{r}$ on system response. $\_r ; \ldots z_{r} ; \_C_{\hat{K}}$; Additional legend elements in Table $2 \ldots \ldots . \ldots . \ldots . \ldots . \ldots 33$

8 Effect of $W_{r}$ on thrust commands. $-u_{T}^{C_{\hat{K}}} \ldots \ldots \ldots \ldots$

9 Adaptive Model Control . . . . . . . . . . . . . . . . . 42

10 Adaptive Model Control for LTI Single Channel Quadcopter . . . . . . . . 45

11 General MRAC Structure . . . . . . . . . . . . . . . . 46

12 MIT Rule . . . . . . . . . . . . . . . . . . . . . . 49

13 Normalized MIT Rule . . . . . . . . . . . . . . . . . . . 51

14 State Feedback Direct MRAC . . . . . . . . . . . . . . 53

15 LTV Percent Difference of Error and Reference . . . . . . . . . . . . . 54 


\section{INTRODUCTION}

Most practical control systems have actuators with limited range and many also exhibit linear parameter-varying (LPV) plants. When not addressed, actuator saturation and parameter variations can result in a significant deviation from nominal performance and worst case, system instability. Some control systems address input saturation by designing the controller such that saturation is avoided altogether. For example, in $[14,13]$, the "positive $\mu$-modification" allows the adaptive system to maintain stable adaptation during saturation events by modifying the reference command to ensure the control signal remains within the saturation limits. While solving the input saturation problem, controllers of this form do not explicitly include anti-windup techniques, which can provide favorable performance over their non-anti-windup contestants. Likewise, these control strategies do not make use of the full actuator range and often lead to conservative designs with suboptimal performance. Alternatively, in [23, 26], rather than avoiding saturation limits, the positive $\mu$-modification is altered to demonstrate anti-windup capabilities in control signal magnitude [23] and rate [26] limited control systems. These methods make use of model reference adaptive control (MRAC) as the nominal controller for a linear time-invariant (LTI) plant. An adaptive, deadzone dependent anti-windup term is included in both the control law and the reference model. The modified reference model contains the additional anti-windup term that alters the desired plant trajectories. When there is no saturation, the anti-windup term has no effect on the controller output, and the ideal reference model is tracked. When saturation is present, the modified reference model is tracked until the antiwindup term returns the plant to ideal reference model tracking. Theoretical development guarantees the convergence of the plant trajectories to the modified reference model trajec- 
tories and eventually to the ideal reference model trajectories. However, the methods are not extended to LPV systems.

Various anti-windup strategies have been developed for LPV systems. In [18], an LPV anti-windup design method is presented that assumes the plant is LTI but includes the feedback of a nonlinear operator that contains both the time-varying parameters and the input nonlinearity. The results from this paper guarantee a single upper bound on the anti-windup $\mathscr{L}_{2}$ performance which may result in conservatism for certain operating conditions. In [5], the one-step anti-windup design paradigm is applied. In this case, $\mathscr{L}_{2}$ performance is only guaranteed for the unsaturated system. During saturation, the performance requirements are relaxed, and only bounded system trajectories are guaranteed. Similarly, [28] applies the one-step anti-windup design which also results in conservative saturated performance. However, this paper takes the unique approach of using a saturation indicator as a scheduling variable for the control system which allows the performance degradation to be smooth when saturation occurs. The two-step anti-windup design is applied to LPV systems in [9]. However, the method presented requires the simultaneous solution of multiple linear matrix inequalities (LMIs) and multiple intermediate steps to construct the anti-windup compensator. In each of these anti-windup designs (except [18]), gain-scheduling techniques are applied. For LPV systems where the parameters can be measured or estimated in real-time, it is known that a gain-scheduled controller will yield higher performance than an LTI or robust controller [16].

Input saturation in adaptive control systems leads to undesirable adaptation of the controller. This issue is addressed in [17] where a binary multiplier is included in the MRAC adaptation laws to suspend adaptation when saturation is present. This modification to MRAC prevents instability and controller windup caused by increasing controller gains but does not specifically include the performance guarantees that anti-windup compensation (AWC) provides. While MRAC and AWC are rather developed fields, research regarding the combination of the two control systems, particularly for LPV systems, is less devel- 
oped. In [3] and [20], MRAC is used with AWC, but the controllers are only developed for LTI plants. In [3], the controller consists of MRAC and an adaptive anti-windup compensator. The anti-windup synthesis is included in the design of MRAC adaptation laws which makes the design appealing. However, the incorporation of AWC in the MRAC control law development results in an anti-windup compensator that is not designed to have optimal $\mathscr{L}_{2}$ performance, which has been demonstrated to be critical for good anti-windup performance in LTI systems [24, 10, 29].

Considering the plant used in this paper, a quadcopter may retrieve packages of unknown mass which results in an overall change to the plant's inertia. Furthermore, a heavy package that challenges the quadcopter's lift capability will likely cause actuator (thrust) saturation, particularly if additional thrust is required to overcome unpredictable disturbances such as wind. It is important to consider that while the quadcopter thrust capabilities could simply be over-designed to easily carry these heavier packages, this would require larger actuators which would increase the overall quadcopter mass. A more powerful quadcopter, while well suited for carrying heavier packages, will expend a significant amount of its battery life to transport its own weight when carrying lighter packages. So, rather than use a heavier and more powerful quadcopter, a control system with an adaptive controller in the two-step anti-windup design procedure is proposed that allows less powerful quadcopters to stably transport these "heavier" packages. With MRAC as the nominal adaptive controller, the unsaturated system maintains stability and desired performance despite parameter variations. During saturation, a novel parameter-dependent anti-windup compensator (PDAWC) maintains stability and optimal $\mathscr{L}_{2}$ performance.

The rest of the paper continues as follows: Section 2 presents common notation used throughout the paper. Following the two-step anti-windup design process, the nominal MRAC controller and PDAWC are designed independently and are subsequently combined in Section 3. In Section 3, a system identification method is also presented to estimate the time-varying inertia parameters. The LPV quadcopter dynamics are developed in Section 4. 
The proposed control system is designed and applied in simulation to the LPV quadcopter in Section 5. Conclusions and future work are presented in Section 6. In Appendix A, different methods of adaptive control are examined. Prior to choosing state feedback direct MRAC as the nominal adaptive controller to be applied to the quadcopter, other adaptive control methods were also considered. Appendix A highlights each of the types of adaptive control explored in this preliminary research period and also provides explanations for why they were not chosen to be the nominal adaptive controller for combination with the PDAWC and subsequent application to the quadcopter in simulation. 


\section{NOTATION}

This paper uses standard notation. A matrix in the real space $m \times n$ is represented by $\mathbb{R}^{m \times n}$. The identity matrix is given by $I_{q} \in \mathbb{R}^{q \times q}$. The saturation function sat $(\cdot): \mathbb{R}^{m} \mapsto \mathbb{R}^{m}$ is defined as

$$
\operatorname{sat}(u):=\left[\operatorname{sat}_{1}\left(u_{1}\right) \ldots \operatorname{sat}_{m}\left(u_{m}\right)\right]^{\prime}
$$

where

$$
\operatorname{sat}_{i}\left(u_{i}\right):=\left\{\begin{array}{l}
\bar{u}_{i} \text { if } \quad u_{i}>\bar{u}_{i} \\
u_{i} \text { if }-\underline{u}_{i} \leq u_{i} \leq \bar{u}_{i} \quad, \bar{u}_{i}, \underline{u}_{i} \geq 0 \\
-\underline{u}_{i} \text { if } \quad u_{i}<-\underline{u}_{i}
\end{array}\right.
$$

The deadzone function $\mathrm{Dz}(\cdot): \mathbb{R}^{m} \mapsto \mathbb{R}^{m}$ is given by

$$
\tilde{u}=\mathrm{Dz}(u):=u-\operatorname{sat}(u)
$$

A discrete vector-valued signal $v(k)$ has $\mathscr{L}_{2}$ norm given by

$$
\|v\|_{2}=\left(\sum_{0}^{\infty}\|v(k)\|^{2}\right)^{1 / 2}
$$

where $\|v(k)\|=\sqrt{v(k)^{\prime} v(k)}$. 


\section{COMBINED MRAC AND PDAWC CONTROL SYNTHESIS}

The control architecture, shown in Fig. 1, includes the PDAWC $\Theta(\rho)$ and nominal controller $K(\rho)$ with general MRAC structure. The MRAC consists of a reference model $G_{r}(s)$ that contains the ideal plant dynamics and an adaptive gain matrix $\bar{K}(\rho)$ that is designed to eliminate deviations between the trajectories of the plant $G(\rho)$ and the ideal trajectories specified by the reference model. The presented architecture is based

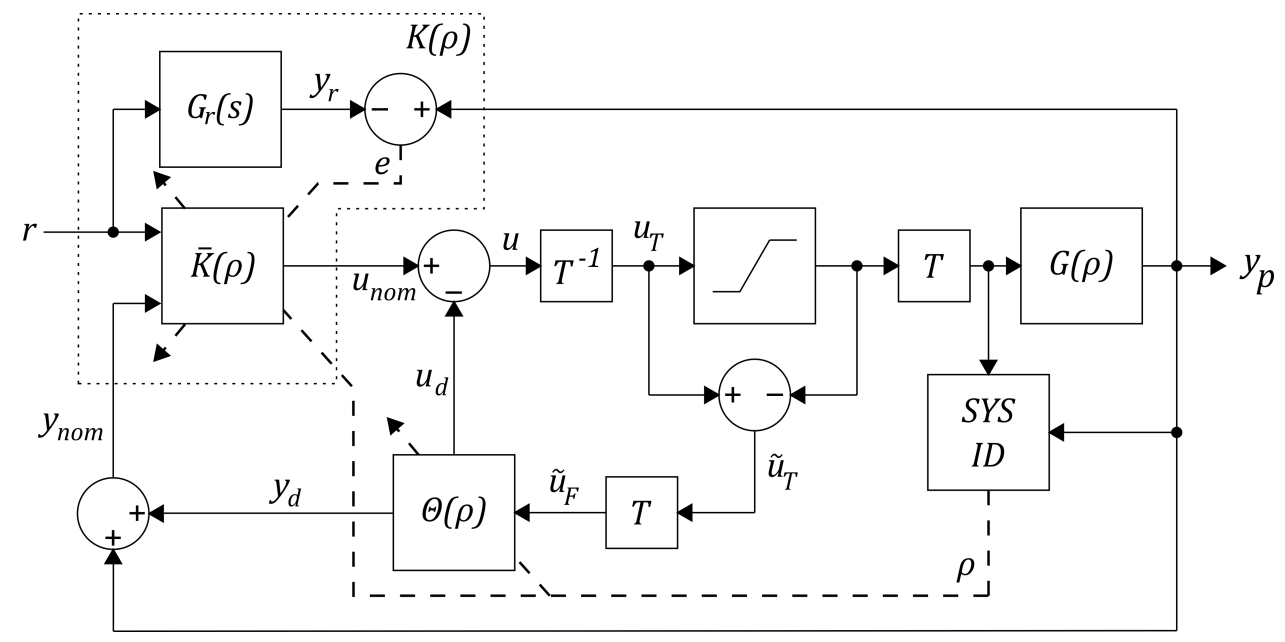

Figure 1: Combined MRAC and PDAWC architecture.

on the general LTI anti-windup design in $[24,10]$ and the LPV anti-windup design in [18]. The architecture presented in Fig. 1 differs from [24, 10], which exclude plant, anti-windup, and nominal controller dependence on $\rho$ and the control allocation matrix $T$. In [18], $T$ is also excluded, but parameter dependence of the plant, anti-windup, and nominal controller is considered in linear fractional transformation form rather than the direct dependence on the parameter estimate $\rho$ as presented in Fig 1. The control allocation matrix $T \in \mathbb{R}^{m \times m}$ is included for application to diagonally structured plants, i.e., $G(\rho)=\operatorname{blockdiag}\left(G_{1}(\rho), \ldots, G_{m}(\rho)\right)$. In Section 4 , the quadcopter is shown to be repre- 
sented with this diagonal structure. For full discussion of the inclusion of $T$ for diagonally structured plants in anti-windup design see $[25,19]$.

Consider the following plant with an LPV input matrix and input constraints

$$
G(\rho) \sim\left\{\begin{array}{l}
\dot{x}_{p}=A_{p} x_{p}+B_{p}(\rho) T \operatorname{sat}\left(u_{T}\right) \\
y_{p}=C_{p} x_{p} \\
z_{p}=C_{p z} x_{p}
\end{array}\right.
$$

where $x_{p} \in \mathbb{R}^{n_{p}}$ is the plant state vector, and $\rho \in \mathbb{R}^{v}$ is the vector of time-varying parameters with elements that are magnitude limited to vary within a set $\mathscr{P}$ defined as $\mathscr{P}=\{\rho$ : $\left.\underline{\rho}_{i} \leq \rho_{i} \leq \bar{\rho}_{i}, i=1,2, \ldots, v\right\}$. The lower and upper bounds for the parameter vector $\rho$ are denoted as $\varrho=\left[\varrho_{1} \ldots \underline{\rho}_{v}\right]$ and $\bar{\rho}=\left[\bar{\rho}_{1} \ldots \bar{\rho}_{v}\right]$, respectively. In Fig. 1, both the nominal controller and PDAWC rely upon the time varying parameters $\rho$. These parameters are determined by system identification which is discussed specifically in Section 3.3. The thrust control signal is $u_{T} \in \mathbb{R}^{m}$. It is assumed all states are available by either estimation or measurement; therefore, $C_{p}=I_{n_{p}}$, and the plant output $y_{p}=x_{p}$. The output $z_{p} \in \mathbb{R}^{n_{r}}$ is the vector of performance variables used in reference tracking. The anti-windup compensator $\Theta(\rho)$ is driven by the signal $\tilde{u}_{F}=T \tilde{u}_{T} \in \mathbb{R}^{m}$, where $\tilde{u}_{T}=u_{T}-\operatorname{sat}\left(u_{T}\right)=\operatorname{Dz}\left(u_{T}\right)$, and emits two signals $u_{d} \in \mathbb{R}^{m}$ and $y_{d} \in \mathbb{R}^{n_{p}}$, which are injected at the output and input of $\bar{K}(\rho)$, respectively. The MRAC gain matrix $\bar{K}(\rho)$ is driven by $y_{\text {nom }} \in \mathbb{R}^{n_{p}}$ and the reference command $r \in \mathbb{R}^{r}$, and it generates the control signal $u_{\text {nom }} \in \mathbb{R}^{m}$. The error between the plant and reference model outputs, $e \in \mathbb{R}^{n_{p}}$, is defined as

$$
e=y_{p}-y_{r}
$$

The MRAC gain matrix $\bar{K}(\rho)$ is designed to adapt such that $y_{p}$ tracks the desired reference model trajectories $y_{r} \in \mathbb{R}^{n_{p}}$, i.e., $e \rightarrow 0$ [1].

In the two-stage anti-windup design procedure, the nominal controller $K(\rho)$ is designed 
without consideration of the input constraints and is assumed to stabilize the closed-loop system in the absence of saturation. A detailed design process and stability analysis of MRAC for LPV plants follows in Section 3.1. The PDAWC $\Theta(\rho)$ is designed to satisfy the following objectives:

1. Remain inactive unless saturation occurs.

2. Ensure closed-loop stability when the control signal $u_{T}$ is saturated.

3. Provide fast recovery to nominal control when saturation ceases.

The first item is guaranteed since $\tilde{u}_{F}=0$ when $u_{T}=\operatorname{sat}\left(u_{T}\right)$. The second item is guaranteed if the PDAWC design is stable, which will shown to be the case as presented in Section 3.2. The remaining item represents anti-windup performance, which, as presented in Section 3.2 , is achieved in the $\mathscr{L}_{2}$ sense.

\subsection{Model Reference Adaptive Control}

As mentioned previously, the two-stage anti-windup design procedure enables the design of the nominal controller without consideration of input constraints. Therefore, in the absence of saturation, the architecture in Fig. 1 reduces to the architecture shown in Fig. 2. Note that since control allocation cancels, the input to the plant (4) is the output $u$ of the nominal control.

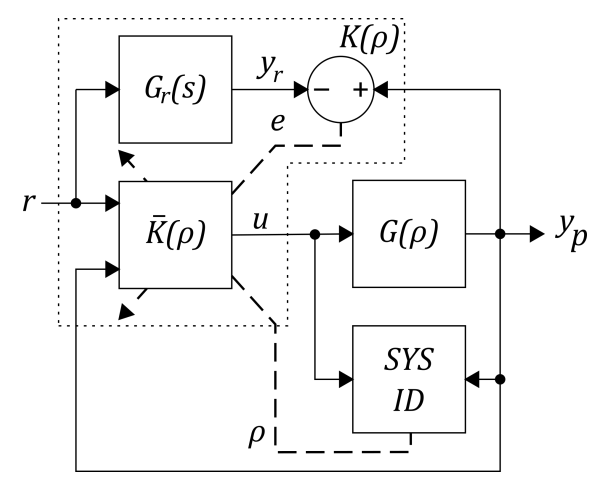

Figure 2: General MRAC system. 
While Fig. 2 represents the general MRAC structure (with added system identification and parameter dependence), the specific MRAC structure utilized in this paper includes integral feedback which is shown in Fig. 3 [15]. This structure ensures zero steady-state reference tracking error by regulating the following integral: $x_{I}=\int e_{r} d t$ where $e_{r}=r-z_{p}$. The nominal controller $K(\rho)$ shown in Fig. 3 will be used in place of the general MRAC $K(\rho)$ in the architecture presented in Fig. 1.

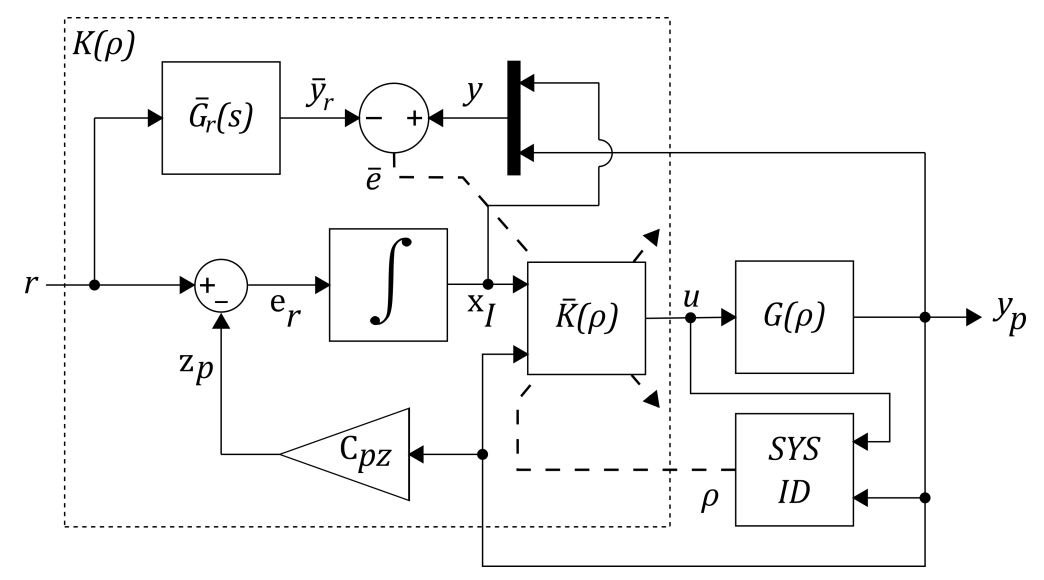

Figure 3: MRAC with Integral Feedback.

By defining $x=\left[x_{I}^{\prime} \quad x_{p}^{\prime}\right]^{\prime} \in \mathbb{R}^{n_{r}+n_{p}}$, a modified version of (4) with open-loop system dynamics containing the additional error states can be written as

$$
\bar{G}(\rho) \sim\left\{\begin{array}{l}
\dot{x}=A x+B(\rho) u+B_{r} r \\
y=C x
\end{array}\right.
$$

where

$$
A=\left[\begin{array}{cc}
0 & -C_{p z} \\
0 & A_{p}
\end{array}\right] \quad B(\rho)=\left[\begin{array}{c}
0 \\
B_{p}(\rho)
\end{array}\right] \quad B_{r}=\left[\begin{array}{c}
I_{n_{r}} \\
0
\end{array}\right] \quad C=\left[\begin{array}{cc}
I_{n_{r}} & 0 \\
0 & C_{p}
\end{array}\right]
$$

Since it is assumed that all states from (4) are available, i.e., $C_{p}=I$, then $C=I$ and $y=x$. 
The reference model, which specifies the ideal performance of (6), is given by

$$
\bar{G}_{r}(s) \sim\left\{\begin{array}{l}
\dot{x}_{r}=A_{r} x_{r}+B_{r} r \\
\bar{y}_{r}=C_{r} x_{r}
\end{array}\right.
$$

where $x_{r} \in \mathbb{R}^{n_{r}+n_{p}}$ is the reference model state vector, $\bar{y}_{r} \in \mathbb{R}^{n_{r}+n_{p}}$ is the reference model output for the integral error structure. Choosing the reference model output matrix such that $C_{r}=C=I$, then $\bar{y}_{r}=x_{r}$. Therefore, the error between the plant and reference model outputs originally defined in (5) is now defined as

$$
\bar{e}=x-x_{r}
$$

If the system (6) were parameter independent, ideal performance could be achieved with a fixed gain controller of the form

$$
u=K^{*} x
$$

which can be designed using pole-placement, LQR, or other modern control methods [15] where $K^{*} \in \mathbb{R}^{m \times n_{r}+n_{p}}$ is the ideal fixed gain matrix. Inserting (10) into (6) with $B(\rho)=B$ yields the ideal closed-loop system dynamics of the LTI plant

$$
\dot{x}=\left(A+B K^{*}\right) x+B_{r} r
$$

To ensure the trajectories of the plant follow the desired trajectories of the reference model, the following matching condition must be met [11]

$$
A_{r}=A+B K^{*}
$$

where $A_{r}$ is Hurwitz and is a part of the state-space representation of the reference model 
in (8). To accommodate for the LPV plant, an adaptive control law is developed such that the system in Fig. 3 is closed-loop stable and (9) asymptotically converges to zero. This is achieved by introducing an adaptive term to the control law [15]

$$
u=\underbrace{\left(\hat{K}(\rho)+K^{*}\right)}_{\bar{K}(\rho)} x=\underbrace{\hat{K}(\rho) x}_{u_{a}}+\underbrace{K^{*} x}_{u_{L}}
$$

where $\hat{K}(\rho) \in \mathbb{R}^{m \times n_{r}+n_{p}}$ is the parameter dependent adaptive gain matrix.

Remark. The control signal (13) contains two terms: the adaptive control signal $u_{a}$ and a control signal for the optimal LTI controller $u_{L}$. The inclusion of $u_{L}$ is not required to prove stable dynamics of $\hat{K}(\rho)$ with the Lyapunov stability analysis that follows. However, by including $u_{L}$ in the control law, the performance at initialization is significantly improved since, upon initialization, $\hat{K}(\rho)$ has no preset value (see (24)). Therefore, if $u_{a}$ is the only control signal, performance is likely to be poor until $\hat{K}(\rho)$ has adapted to ensure $\bar{e}$ is either small or zero. When $u_{L}$ is present, the closed-loop system is initialized with the same closed-loop poles as the reference model, and the adaptation of $\hat{K}(\rho)$ is only necessary to account for transients or parameter changes that cause deviations between the plant and reference model outputs.

To determine the dynamics of the adaptive gain matrix $\hat{K}(\rho)$ that ensure $\bar{e} \rightarrow 0$, the state equation of (6) can be equivalently written as

$$
\dot{x}=A_{r} x+B(\rho)\left(u+B^{+}(\rho)\left(A-A_{r}\right) x\right)+B_{r} r
$$

where $B^{+}(\rho)=B^{\prime}(\rho)\left(B(\rho) B^{\prime}(\rho)\right)^{-1}$ denotes the Moore-Penrose inverse. By defining $\mathscr{K}(\rho)=-B^{+}(\rho)\left(A-A_{r}\right)$, then if the adaptive gain matrix $\bar{K}(\rho)$ can converge to $\mathscr{K}(\rho)$, then the control law (13) results in plant dynamics (14) that are identical to the reference model dynamics (8) [2]. Substituting $\mathscr{K}(\rho)$ and (13) into (14), the closed-loop dynamics 
become

$$
\dot{x}=A_{r} x+B(\rho) \Delta \bar{K}(\rho) x+B_{r} r
$$

where $\Delta \bar{K}(\rho)=\bar{K}(\rho)-\mathscr{K}(\rho)$. The dynamics of (9) can be obtained by subtracting the reference model state equation in (8) from (15)

$$
\begin{aligned}
\dot{\bar{e}} & =\dot{x}-\dot{x}_{r} \\
& =A_{r} x+B(\rho) \Delta \bar{K}(\rho) x-A_{r} x_{r} \\
& =A_{r} \bar{e}+B(\rho) \Delta \bar{K}(\rho)
\end{aligned}
$$

Now, consider a Lyapunov function candidate

$$
V(x, t)=\bar{e}^{\prime} P \bar{e}+\operatorname{trace}\left(\Delta \bar{K}(\rho) \Gamma_{K}^{-1} \Delta \bar{K}^{\prime}(\rho)\right)
$$

where $\Gamma_{K}>0$ is the diagonal adaptation rate matrix and $P=P^{\prime}>0$ is the unique solution to the algebraic Lyapunov equation

$$
P A_{r}+A_{r}^{\prime} P=-\mathscr{Q}_{K}
$$

for any $\mathscr{Q}_{K}=\mathscr{Q}_{K}^{\prime}>0$. The derivative of $V(x, t)$ along its trajectories is

$$
\dot{V}(x, t)=\bar{e}^{\prime} P \dot{\bar{e}}+\dot{\bar{e}}^{\prime} P \bar{e}+2 \operatorname{trace}\left(\Delta \bar{K}(\rho) \Gamma_{K}^{-1} \dot{\hat{K}}^{\prime}(\rho)\right)
$$

Substituting (18) into (21),

$$
\dot{V}(x, t)=\bar{e}^{\prime} \underbrace{\left(P A_{r}+A_{r}^{\prime} P\right)}_{-\mathscr{Q}_{K}} \bar{e}+2 \underbrace{\bar{e}^{\prime} P B(\rho)}_{a^{\prime}} \underbrace{\Delta \bar{K}(\rho) x}_{b}+2 \operatorname{trace}\left(\Delta \bar{K}(\rho) \Gamma_{K}^{-1} \dot{\hat{K}}^{\prime}(\rho)\right)
$$

where co-dimensional vectors $a$ and $b$ satisfy the trace identity $a^{\prime} b=\operatorname{trace}\left(b a^{\prime}\right)$. The deriva- 
tive of the Lyapunov function can then be written as

$$
\dot{V}(x, t)=-\bar{e}^{\prime} \mathscr{Q}_{K} \bar{e}+2 \operatorname{trace}\left(\Delta \bar{K}(\rho)\left(x \bar{e}^{\prime} P B(\rho)+\Gamma_{K}^{-1} \dot{\hat{K}}^{\prime}(\rho)\right)\right)
$$

If the adaptive law is chosen as

$$
\dot{\hat{K}}^{\prime}(\rho)=-\Gamma_{K} x \bar{e}^{\prime} P B(\rho)
$$

then $\dot{V}(x, t)$ becomes globally negative semi-definite

$$
\dot{V}(x, t)=-\bar{e}^{\prime} \mathscr{Q}_{K} \bar{e} \leq 0
$$

This condition guarantees the closed-loop dynamics of $\bar{e}$ are uniformly stable. Additionally, the uniform stability of $\dot{\bar{e}}$ ensures that the terms $\bar{e}$ and $\hat{K}(\rho)$, which are contained in $\dot{V}(x, t)$, are uniformly bounded. With the bounded reference command $r$ and $A_{r}$, which is Hurwitz, the reference state $x_{r}$ and its first time derivative are bounded from (8). Because $\bar{e}$ and $x_{r}$ are bounded, then from (9), $x$ is bounded, which ensures $x_{p}$ is also bounded. The control input $u$ is bounded as a result of the boundedness of $\hat{K}(\rho), x_{p}, \bar{e}$, and $r$. Boundedness of $u$ results in bounded $\dot{x}$ as well (6). Since $\dot{x}$ and $\dot{x}_{r}$ are bounded, then from (16) $\dot{\bar{e}}$ is also bounded. Because both $\bar{e}$ and $\dot{\bar{e}}$ are bounded, the second derivative of $V$, given by

$$
\ddot{V}(x, t)=-2 \bar{e}^{\prime} \mathscr{Q}_{K} \dot{\bar{e}}
$$

is also bounded, thus $\dot{V}(x, t)$ is uniformly continuous. Additionally, $V$ is lower bounded by zero and $\dot{V}(x, t) \leq 0$. With these conditions, Barbalat's lemma can be applied with use of the immediate corollary:

Lemma 1.1 ("Lyapunov-Like Lemma") If a scalar function $V(x, t)$ satisfies the following conditions 
- $V(x, t)$ is lower bounded

- $\dot{V}(x, t)$ is negative semi-definite

- $\dot{V}(x, t)$ is uniformly continuous in time

then $\dot{V}(x, t) \rightarrow 0$ as $t \rightarrow \infty[21]$.

Therefore, $\bar{e}$ globally, uniformly, and asymptotically converges to the origin.

\subsection{Parameter-Dependent Anti-Windup Compensation}

The two-step anti-windup method parameterizes the anti-windup compensator $\Theta(\rho)$ in terms of a transfer function $M(\rho)$ and a copy of the plant $G(\rho)$. Under this parameterization, the system depicted in Fig. 1 is equivalent to the system in Fig. 4. The equivalent

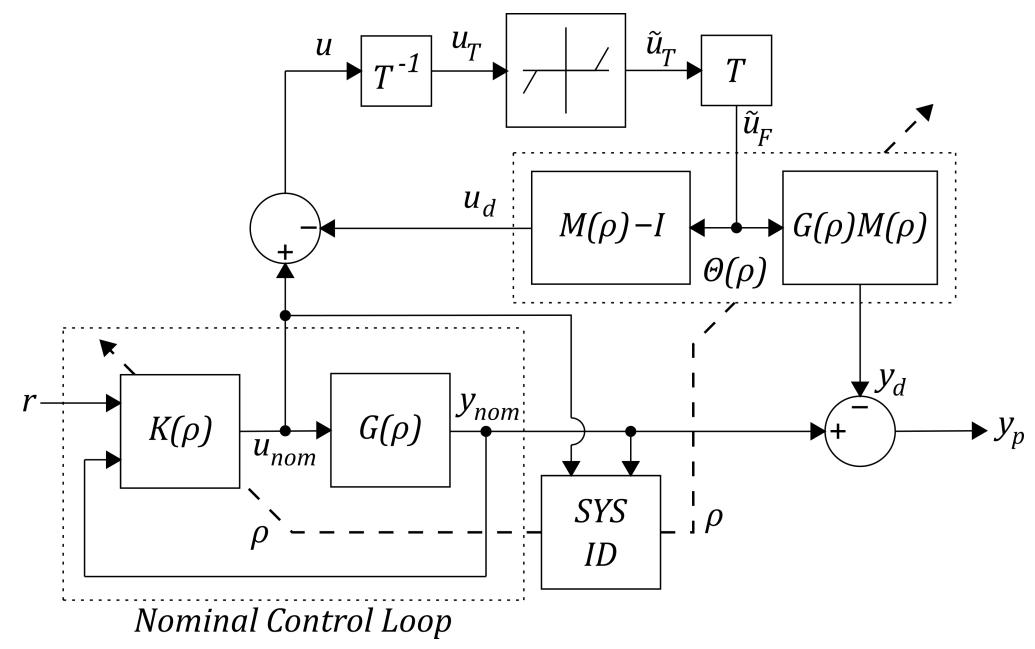

Figure 4: Equivalent representation of Fig. 1 when $\Theta(\rho)$ is parameterized by transfer functions $G(\rho)$ and $M(\rho)$

structure illustrates the two-step anti-windup design process, i.e., that stability analysis and design for the nominal control loop and the anti-windup compensation loop can be performed separately. Furthermore, Fig. 4 illuminates that minimizing the saturated system's deviation from nominal performance can achieved by the minimization of the mapping $\mathscr{T}_{\text {aw }}(\rho): u_{\text {nom }} \mapsto y_{d}$. 
The objectives of anti-windup compensator design presented in the introduction of this section will be achieved by choosing $M(\rho)$ as part of the right co-prime factorization of $G(\rho)=N(\rho) M(\rho)^{-1}$ where $M(\rho), N(\rho) \in \mathscr{R} \mathscr{H}_{\infty}[6]$. With this choice of $M(\rho)$, the fullorder anti-windup compensator

$$
\Theta(\rho)=\left[\begin{array}{c}
M(\rho)-I \\
N(\rho)
\end{array}\right]
$$

has order $\mathbb{R}^{n_{p}}$ and shares the same state space as the plant (4) [24].

To build off the extensive work in [10] regarding discrete anti-windup design, the antiwindup compensator presented in this paper is also designed to be discrete. Additionally, as a result of the discrete nature of the system identification technique used in Section 3.3, $\rho$ is not known continuously but as a discrete vector updated at each time step $k$ i.e. $\rho=\rho(k \Delta t)$ where $\Delta t$ is the discrete update step size of the system identification method. Thus, the use of a discrete anti-windup compensator pairs well with the use of discrete estimates of $\rho$. The discrete anti-windup compensator is designed using the discrete counterpart of the continuous plant $G(\rho)(4)$.

$$
G_{z}(\rho) \sim \begin{cases}x_{z}(k+1) & =A_{z} x_{z}(k)+B_{z}(\rho) T \operatorname{sat}\left(u_{T}(k)\right) \\ y_{z}(k) & =C_{z} x_{z}(k)\end{cases}
$$

where $x_{z}(k) \in \mathbb{R}^{n_{p}}$ is the discrete plant state vector, $u_{T}(k) \in \mathbb{R}^{m}$ is the discrete thrust signal and $y_{z}(k) \in \mathbb{R}^{n_{p}}$ is the discrete plant output. The discrete state space matrices $A_{z}, B_{z}(\rho)$, and $C_{z}$ are determined by applying the zero-order hold ( $\left.\mathrm{ZOH}\right)$ method to (4) [8]. The co-prime 
factors of $G_{z}(\rho)$ to be used in (27) are given by

$$
\begin{aligned}
& M(\rho)=\left[\begin{array}{cc}
A_{z}+B_{z}(\rho) F(\rho) & B_{z}(\rho) \\
F(\rho) & I
\end{array}\right] \\
& N(\rho)=\left[\begin{array}{cc}
A_{z}+B_{z}(\rho) F(\rho) & B_{z}(\rho) \\
C_{z} & 0
\end{array}\right]
\end{aligned}
$$

Substituting (29) and (30) into (27), the state-space representation of $\Theta(\rho)$ is

$$
\Theta(\rho) \sim \begin{cases}x_{a}(k+1) & =\left(A_{z}+B_{z}(\rho) F(\rho)\right) x_{a}(k)+B_{z}(\rho) \tilde{u}_{F}(k) \\ u_{d}(k) & =F(\rho) x_{a}(k) \\ y_{d}(k) & =C_{z} x_{a}(k)\end{cases}
$$

where $x_{a}(k) \in \mathbb{R}^{n_{p}}$ is the anti-windup state and $\tilde{u}_{F}(k)=T \tilde{u}_{T}(k)=T \operatorname{Dz}\left(u_{T}(k)\right) \in \mathbb{R}^{m}$. With the structure of $\Theta(\rho)$ chosen, the remaining task is to calculate the parameter dependent anti-windup gain $F(\rho) \in \mathbb{R}^{m \times n_{p}}$ such that global stability is achieved and $\left\|\mathscr{T}_{a w}(\rho)\right\|_{2}$ is minimized where

$$
\left\|y_{d}(k)\right\|_{2} \leq\left\|\mathscr{T}_{\text {aw }}(\rho)\right\|_{2}\left\|u_{\text {nom }}(k)\right\|_{2}
$$

Theorem 1 . If $K(\rho)$ stabilizes $G(\rho) \forall \rho \in \mathscr{P}$ without consideration of the input nonlinearity, there exists a discrete full order anti-windup compensator $\Theta(\rho)$ which achieves the anti-windup design objectives if for a given $\rho$ there exist matrices $Q(\rho)>0, U(\rho)=$ $\operatorname{diag}\left(\mu_{1}(\rho), \ldots, \mu_{m}(\rho)\right)>0, L(\rho) \in \mathbb{R}^{m \times n}$ and a positive real scalar $\gamma(\rho)$ such that the $L M I$ 
(33) is satisfied.

$$
\left[\begin{array}{ccccc}
-Q(\rho) & -L^{\prime}(\rho) & 0 & Q(\rho) A_{z}^{\prime}+L^{\prime}(\rho) B_{z}^{\prime}(\rho) & Q(\rho) C_{z}^{\prime} \\
\star & -2 U(\rho) & I_{m} & U(\rho) B_{z}^{\prime}(\rho) & 0 \\
\star & \star & -\gamma(\rho) I_{m} & 0 & 0 \\
\star & \star & \star & -Q(\rho) & 0 \\
\star & \star & \star & \star & -\gamma(\rho) I_{m}
\end{array}\right]<0
$$

For each $\rho$, the solution to (33) yields an $F(\rho)$ that achieves $\left\|\mathscr{T}_{a w}(\rho)\right\|_{2}<\gamma(\rho)$ and is given by $F(\rho)=L(\rho) Q^{-1}(\rho)$.

Proof. A Lyapunov function candidate is chosen as $V\left(x_{a}(k)\right)=x_{a}^{\prime}(k) P(\rho) x_{a}(k)>0$ where $P(\rho) \in \mathbb{R}^{n_{p}}$ is a real symmetric positive definite matrix. The Lyapunov difference is given by $\Delta V\left(x_{a}(k)\right):=V\left(x_{a}(k+1)\right)-V\left(x_{a}(k)\right)$. Since the system in Fig. 4 is equivalent to the system in Fig. 1, then if the inequality

$$
\Delta V\left(x_{a}(k)\right)<\gamma^{2}(\rho)\left\|u_{n o m}(k)\right\|^{2}-\left\|y_{d}(k)\right\|^{2}
$$

is satisfied then the system (31) is is finite-gain $\mathscr{L}_{2}$ stable and $\left\|\mathscr{T}_{a w}(\rho)\right\|_{2}<\gamma(\rho)$ [12].

The deadzone $\mathrm{Dz}(\cdot): \mathbb{R}^{m} \mapsto \mathbb{R}^{m}$ inhabits the sector $[0, I][12]$. Thus, the following sector condition is satisfied for all diagonal $W(\rho)>0$

$$
\tilde{u}_{T}(k)^{\prime} W(\rho)\left(u(k)-\tilde{u}_{T}(k)\right) \geq 0
$$

where $u(k)=u_{\text {nom }}(k)-u_{d}(k)$. However, since the PDAWC input is $\tilde{u}_{F}(k)$, then if diagonal $Y(\rho)=\left(T^{-1}\right)^{\prime} W(\rho) T^{-1}$ exists, then the following inequality is satisfied

$$
\tilde{u}_{F}(k)^{\prime} Y(\rho)\left(u(k)-\tilde{u}_{F}(k)\right) \geq 0
$$

As shown in [19], diagonal positive definite matrices $W(\rho), Y(\rho) \in \mathbb{R}^{m}$, such that 
$\left(T^{-1}\right)^{\prime} W(\rho) T^{-1}=Y(\rho)$, do exist for the $T$ matrix of the quadcopter presented in Section

4. Therefore,

$$
\begin{aligned}
& \Delta V\left(x_{a}(k)\right)+\left\|y_{d}(k)\right\|^{2}-\gamma^{2}(\rho)\left\|u_{n o m}(k)\right\|^{2} \\
< & \Delta V\left(x_{a}(k)\right)+\left\|y_{d}(k)\right\|^{2}-\gamma^{2}(\rho)\left\|u_{n o m}(k)\right\|^{2}+2 \tilde{u}_{F}(k)^{\prime} Y(\rho)\left(u_{n o m}(k)-u_{d}(k)-\tilde{u}_{F}(k)\right)<0
\end{aligned}
$$

To begin formulation of the LMI (33), substitute $x_{a}(k+1), x_{a}(k), u_{d}(k), y_{d}(k)$ into (37)

$$
\left[\begin{array}{c}
x_{a}(k) \\
\tilde{u}_{F}(k) \\
u_{\text {nom }}(k)
\end{array}\right]^{\prime}\left[\begin{array}{ccc}
V_{11} & \left(A_{z}+B_{z}(\rho) F(\rho)\right)^{\prime} P(\rho) B_{z}(\rho)-2 F^{\prime}(\rho) Y(\rho) & 0 \\
\star & -2 Y(\rho)+B_{z}^{\prime}(\rho) P(\rho) B_{z}(\rho) & Y(\rho) \\
\star & \star & -\gamma^{2}(\rho) I_{m}
\end{array}\right]\left[\begin{array}{c}
x_{a}(k) \\
\tilde{u}_{F}(k) \\
u_{\text {nom }}(k)
\end{array}\right]
$$

where

$$
V_{11}=\left(A_{z}+B_{z}(\rho) F(\rho)\right)^{\prime} P(\rho)\left(A_{z}+B_{z}(\rho) F(\rho)\right)-P(\rho)+C_{z}^{\prime} C_{z}
$$

The standard Schur complement and the congruence transformation $\mathscr{C}=\operatorname{diag}\left(P^{-1}(\rho) Y^{-1}(\rho) I I I\right)$ are applied to (38). Then, using the relationships $U(\rho)=$ $Y^{-1}(\rho), Q(\rho)=P^{-1}(\rho), L(\rho)=F(\rho) Q(\rho)$, and $L^{\prime}(\rho)=Q(\rho) F^{\prime}(\rho)$, (38) becomes the LMI governing global stability of $\Theta(\rho)$ as expressed in (33).

\subsubsection{Local Stability}

The quadcopter for which this anti-windup compensator is to be applied in Section 5 is open-loop marginally stable - all of the poles of the linearized system lie at the origin of the complex plane. Therefore, only local stability can be achieved by which it is assumed that the state $x_{a}(k)$ attains values only within a certain compact region of the state space. This means that the input to the deadzone $u_{T}(k)$ attains values only below a certain level, i.e., if all $u_{T, i}(k)$ satisfy $-\beta_{i} \underline{\mathrm{u}}_{T, i} \leq u_{T, i}(k) \leq \beta_{i} \bar{u}_{T, i}$, where $\beta_{i}>1 \forall i$, then $\mathrm{Dz}_{i}\left(u_{T, i}(k)\right)$ remains 
below the gradient

$$
\alpha_{i}:=\frac{\beta_{i}-1}{\beta_{i}}<1
$$

Therefore, the deadzone is constrained to the narrower sector bound Sector $[0, \mathscr{A}]$ where $\mathscr{A}:=\operatorname{diag}\left(\alpha_{1}, \ldots, \alpha_{m}\right)$ and $\alpha_{i} \in(0,1)$. Consequently,

$$
\tilde{u}_{F}(k)^{\prime} Y(\rho)\left[\mathscr{A} u(k)-\tilde{u}_{F}(k)\right] \geq 0
$$

where, again $u(k)=u_{\text {nom }}(k)-u_{d}(k)$ and $Y(\rho)=\left(T^{-1}\right)^{\prime} W(\rho) T^{-1}$. Following the same process for developing (33) from (37) with the slight variation of replacing (36) with (40), the parameter dependent LMI (41) is generated with an optimal solution for each $\rho$ given by $F(\rho)$ in (31) that achieves $\left\|\mathscr{T}_{a w}(\rho)\right\|_{2}<\gamma(\rho)$ for marginally stable and unstable plants and is given by $F(\rho)=L(\rho) Q^{-1}(\rho)$.

$$
\left[\begin{array}{ccccc}
-Q(\rho) & -L^{\prime}(\rho) \mathscr{A} & 0 & Q(\rho) A_{z}^{\prime}+L^{\prime}(\rho) B_{z}^{\prime}(\rho) & Q(\rho) C_{z}^{\prime} \\
\star & -2 U(\rho) & \mathscr{A} & U(\rho) B_{z}^{\prime}(\rho) & 0 \\
\star & \star & -\gamma(\rho) I_{m} & 0 & 0 \\
\star & \star & \star & -Q(\rho) & 0 \\
\star & \star & \star & \star & -\gamma(\rho) I_{m}
\end{array}\right]<0
$$

\subsection{System Identification}

The elements of $\rho$ are estimated by a linear input-output relationship in the system dynamics given by

$$
d(k)=\hat{\rho}_{d}(k) \phi(k)
$$

where $d(k) \in \mathbb{R}^{v}$ is the vector of output variables, $\hat{\rho}_{d}(k)$ is the matrix containing the estimates of $\rho$, and $\phi(k) \in \mathbb{R}^{v}$ is the regressor (input) vector.

To account for changing parameters, a real-time recursive least-squares (RLS) system identification algorithm with exponential forgetting is utilized [4]. This identifies time- 
varying parameters by exponentially discounting the weighting of older data $[1,7]$. The algorithm is applied to the set of linear equations (42) to estimate $\rho(k \Delta t)$ which is needed for both $\hat{K}(\rho)$ from MRAC and the PDAWC $\Theta(\rho)$. The RLS algorithm operates at time steps $k$ and minimizes the following cost function

$$
\mathscr{E}(\rho, k)=\frac{1}{2} \sum_{k=1}^{N} \lambda^{N-k}\left(d(k)-\hat{\rho}_{d}(k) \phi(k)\right)^{2}
$$

where $0<\lambda<1$ is the forgetting factor. The least squares criteria (43) is minimized through implementation of the following recursive equations:

$$
\begin{gathered}
X(k)=\frac{R(k-1) \phi(k)}{\lambda+\phi^{\prime}(k) R(k-1) \phi(k)} \\
\varepsilon(k)=d(k)-\hat{\rho}_{d}(k-1) \phi(k) \\
\hat{\rho}_{d}(k)=\hat{\rho}_{d}(k-1)+X(k) \varepsilon(k) \\
R(k)=\frac{1}{\lambda}\left(R(k-1)-X(k) \phi^{\prime}(k) R(k-1)\right)
\end{gathered}
$$

where $\varepsilon(k)$ is the residual (estimation error). The recursive aspect of these equations requires the initialization of $R(0)=\delta I_{v}$ where $\delta$ is an arbitrary constant. The initial parameter estimate $\hat{\rho}_{d}(0)$ can be initialized to the null vector unless the initial values of $\rho$ are known a priori. From (44c) it can be seen that the current parameter estimate $\hat{\rho}_{d}(k)$ is achieved by an adjustment of the previous estimate which is proportional to the estimation error. 


\section{SYSTEM DYNAMICS}

Fig. 5 represents a quadcopter with time-varying inertia parameters and a body-fixed coordinate frame $\Phi_{1} \equiv\left\{Q ; \vec{i}_{k}\right\}$ aligned along the propeller axes. The quadcopter is moving in space relative to the inertial frame $\Phi_{0} \equiv\left\{E ; \vec{I}_{k}\right\}$. The rotational transformation matrix $R_{10}$ transforms the motion from $\Phi_{0}$ to $\Phi_{1}$ using the $3,2,1$, rotation sequence

$$
R_{10}=\left[\begin{array}{ccc}
c \theta_{3} c \theta_{2} & c \theta_{3} s \theta_{2} s \theta_{1}-s \theta_{3} c \theta_{1} & c \theta_{3} s \theta_{2} c \theta_{1}+s \theta_{3} s \theta_{1} \\
s \theta_{3} c \theta_{2} & s \theta_{3} s \theta_{2} s \theta_{1}+c \theta_{3} c \theta_{1} & s \theta_{3} s \theta_{2} c \theta_{1}-c \theta_{3} s \theta_{1} \\
-s \theta_{2} & c \theta_{2} s \theta_{1} & c \theta_{2} c \theta_{1}
\end{array}\right]
$$

where $c$ and $s$ denote cos and sin functions, respectively [22]. Each propeller generates a thrust $u_{T, i}$ which acts only in the $-\vec{i}_{3}$ direction and is constrained by the saturation function (1).

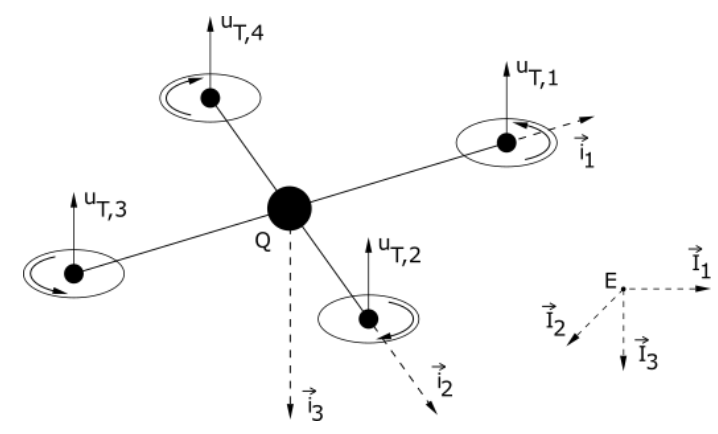

Figure 5: Quadcopter Model.

Translational motion in $\Phi_{1}$ follows the equation

$$
\ddot{x}=\frac{1}{m(t)} F-\dot{\theta} \times \dot{x}+R_{10} \vec{g}
$$


where $x=\left[\begin{array}{lll}x_{1} & x_{2} & x_{3}\end{array}\right]^{\prime}$ and $\theta=\left[\begin{array}{lll}\theta_{1} & \theta_{2} & \theta_{3}\end{array}\right]^{\prime}$ are the translational and rotational degreesof-freedom, respectively, $m(t)$ is the quadcopter mass, $F=\left[\begin{array}{lll}F_{1} & F_{2} & F_{3}\end{array}\right]^{\prime}$ are body forces in each coordinate direction, and $\vec{g}=\left[\begin{array}{lll}0 & 0 & g\end{array}\right]^{\prime}$ where $g$ is the gravitational constant. Since the quadcopter has four unidirectional actuators, only four of the six degrees-of-freedom are chosen to be directly controlled: $x_{3}, \theta_{1}, \theta_{2}$ and $\theta_{3}$, while the remaining degrees-of-freedom are indirect results from these controlled degrees-of-freedom. Therefore, considering only the translational equation of motion along the $\overrightarrow{i_{3}}$ axis:

$$
\ddot{x}_{3}=\frac{F_{3}}{m(t)}+\dot{\theta}_{2} \dot{x}_{1}-\dot{\theta}_{1} \dot{x}_{2}+g \cos \left(\theta_{1}\right) \cos \left(\theta_{2}\right)
$$

Rotational motion of the vehicle is governed by

$$
\ddot{\theta}=J(t)^{-1}(M-\dot{\theta} \times J(t) \dot{\theta})
$$

where $J(t)$ is the mass moment of inertia tensor, and $M=\left[\begin{array}{lll}M_{1} & M_{2} & M_{3}\end{array}\right]^{\prime}$ are the body moments about each coordinate axis in $\Phi_{1}$. Assuming $\Phi_{1}$ is aligned with the principal inertial axes of the quadcopter, $J(t)$ is diagonal

$$
J(t)=\operatorname{diag}\left(J_{11}(t), J_{22}(t), J_{33}(t)\right)
$$

and the rotational equations of motion explicitly are

$$
\begin{aligned}
& \ddot{\theta}_{1}=\frac{1}{J_{11}(t)}\left(M_{1}+\dot{\theta}_{3} J_{22}(t) \dot{\theta}_{2}-\dot{\theta}_{2} J_{33}(t) \dot{\theta}_{3}\right) \\
& \ddot{\theta}_{2}=\frac{1}{J_{22}(t)}\left(M_{2}+\dot{\theta}_{1} J_{33}(t) \dot{\theta}_{3}-\dot{\theta}_{3} J_{11}(t) \dot{\theta}_{1}\right) \\
& \ddot{\theta}_{3}=\frac{1}{J_{33}(t)}\left(M_{3}+\dot{\theta}_{2} J_{11}(t) \dot{\theta}_{1}-\dot{\theta}_{1} J_{22}(t) \dot{\theta}_{2}\right)
\end{aligned}
$$

The control allocation matrix, i.e. the transformation from the propeller thrust 
$u_{T}=\left[\begin{array}{llll}u_{T, 1} & u_{T, 2} & u_{T, 3} & u_{T, 4}\end{array}\right]^{\prime}$ to the body force and moments on the vehicle, is

$$
\left[\begin{array}{l}
F_{3} \\
M_{1} \\
M_{2} \\
M_{3}
\end{array}\right]=\underbrace{\left[\begin{array}{cccc}
-1 & -1 & -1 & -1 \\
0 & -h & 0 & h \\
h & 0 & -h & 0 \\
\tau_{m} & -\tau_{m} & \tau_{m} & -\tau_{m}
\end{array}\right]}_{T} \operatorname{sat}\left(u_{T}\right)
$$

where the half-span, $h$, is the distance from the quadcopter's center of gravity to the each propeller, and $\tau_{m}$ is the torque generated by the rotation of the each propeller. The diagonal of the inertia tensor $J(t)$ can be augmented with the vehicle mass to produce

$$
J_{a}(t)=\operatorname{diag}\left(m(t), J_{11}(t), J_{22}(t), J_{33}(t)\right)
$$

Combining (46), (49), (50), and (51) and using (52) and (53), the quadcopter can be defined by the following equations:

$$
\left[\begin{array}{c}
\ddot{x}_{3} \\
\ddot{\theta}_{1} \\
\ddot{\theta}_{2} \\
\ddot{\theta}_{3}
\end{array}\right]=\underbrace{\left[\begin{array}{c}
\dot{\theta}_{2} \dot{x}_{1}-\dot{\theta}_{1} \dot{x}_{2}+g \cos \left(\theta_{1}\right) \cos \left(\theta_{2}\right) \\
\left(\dot{\theta}_{3} J_{22}(t) \dot{\theta}_{2}-\dot{\theta}_{2} J_{33}(t) \dot{\theta}_{3}\right) / J_{11}(t) \\
\left(\dot{\theta}_{1} J_{33}(t) \dot{\theta}_{3}-\dot{\theta}_{3} J_{11}(t) \dot{\theta}_{1}\right) / J_{22}(t) \\
\left(\dot{\theta}_{2} J_{11}(t) \dot{\theta}_{1}-\dot{\theta}_{1} J_{22}(t) \dot{\theta}_{2}\right) / J_{33}(t)
\end{array}\right]}_{f\left(x_{p}\right)}+J_{a}(t)^{-1} T \operatorname{sat}\left(u_{T}\right)
$$

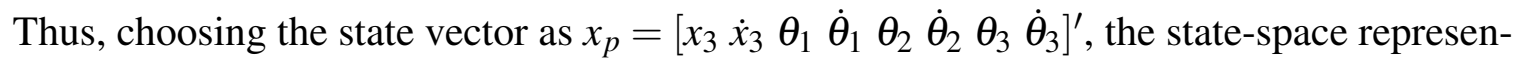
tation of (54) is

$$
\dot{x}_{p}=\underbrace{\left(I_{4} \otimes\left[\begin{array}{ll}
0 & 1 \\
0 & 0
\end{array}\right]\right)}_{A_{p}} x_{p}+\underbrace{\left(J_{a}(t)^{-1} \otimes\left[\begin{array}{l}
0 \\
1
\end{array}\right]\right)}_{B_{p}(\rho)} T \operatorname{sat}\left(u_{T}\right)+f\left(x_{p}\right) \otimes\left[\begin{array}{l}
0 \\
1
\end{array}\right]
$$


where $\otimes$ denotes the Kronecker product, $\rho=\rho(t)$ is the time-varying parameter vector

$$
\rho=\left[\begin{array}{llll}
\frac{1}{m(t)} & \frac{1}{J_{11}(t)} & \frac{1}{J_{22}(t)} & \frac{1}{J_{33}(t)}
\end{array}\right]
$$

$y_{p}=x_{p}$ is the output, and the tracked output states are the position states

$$
z_{p}=\left[\begin{array}{llll}
x_{3} & \theta_{1} & \theta_{2} & \theta_{3}
\end{array}\right]^{\prime}
$$

When the dynamic equations (54) are linearized about the hover operating point, (55) reduces to four decoupled double integrator systems [19]

$$
\dot{x}_{p}=A_{p} x_{p}+B_{p}(\rho) T \operatorname{sat}\left(u_{T}\right)
$$

While the nonlinear dynamics (54) will be used to simulate the quadcopter, the linearized dynamics (58) are used to design the MRAC and PDAWC controllers. 


\section{CONTROL SYSTEM DESIGN AND SIMULATIONS}

To evaluate the performance of the combined MRAC and PDAWC control system, it is applied to an LPV quadcopter with dynamics described by (54) and parameters defined in Table 1. Following from [19], the parameters listed in Table 1 were determined experimentally for a real quadcopter UAV using a mass moment of inertia test rig and force measurement stand. Using parameters of a real quadcopter, the simulation results better approximate the true flight behavior expected in a physical experiment. For comparison, two control systems are designed and applied to the same quadcopter in simulation. The first control system, denoted $C_{\hat{K}, \Theta}$, is the combined MRAC and PDAWC control system depicted in Fig. 1 where the nominal controller $K(\rho)$ implements integral control as shown in Fig. 3. The second control system uses MRAC without any anti-windup compensation, and it is denoted as $C_{\hat{K}}$. The structure of the $C_{\hat{K}}$ control system follows Fig. 3 with saturation and control allocation included at the plant input. Thus, rather than the plant input being $u$ as shown in Fig. 3, the plant input is actually $T$ sat $\left(T^{-1} u\right)$ when implemented in simulation. Both $C_{\hat{K}, \Theta}$ and $C_{\hat{K}}$ are applied to the LPV quadcopter to demonstrate the performance advantage of the proposed parameter-dependent anti-windup compensator when compared to the adaptive control strategy without anti-windup compensation. 
Table 1: Experimentally determined quadcopter parameters.

\begin{tabular}{cccc}
\hline \hline Variable Name & Variable & Value & Units \\
\hline Half-span & $h$ & 0.305 & $\mathrm{~m}$ \\
Gyroscopic moment & $\tau_{m}$ & 0.0278 & $\mathrm{~m}$ \\
Thrust range & {$\left[\underline{\mathrm{u}}_{T, i} \bar{u}_{T, i}\right]$} & {$[04.2]$} & $\mathrm{N}$ \\
Gravitational constant & $g$ & 9.81 & $\mathrm{~m} / \mathrm{s}^{2}$ \\
Initial mass & $m_{o}$ & 1.15 & $\mathrm{~kg}$ \\
Initial mass moment of inertia & $J_{11, o}$ & 0.026 & $\mathrm{~kg} \cdot \mathrm{m}^{2}$ \\
Initial mass moment of inertia & $J_{22, o}$ & 0.026 & $\mathrm{~kg} \cdot \mathrm{m}^{2}$ \\
Initial mass moment of inertia & $J_{33, o}$ & 0.050 & $\mathrm{~kg} \cdot \mathrm{m}^{2}$ \\
\hline \hline
\end{tabular}

The simulations that follow involve lifting a payload with mass $m_{p}$, which is assumed to be unknown. The simulated payload mass, $m_{p}=0.45 \mathrm{~kg}$, is about $40 \%$ of the original vehicle mass $\left(m_{o}\right)$ and is about $80 \%$ of the quadcopter's total payload carrying capacity $(0.56 \mathrm{~kg})$. The payload is assumed to be located at the vehicle's center of mass.

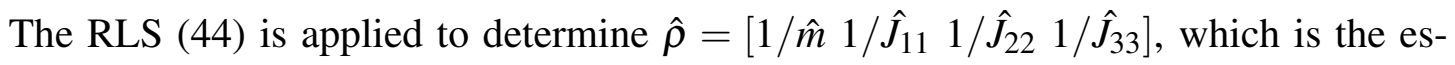
timate of (56). When the quadcopter dynamics (54) are linearized about the hover point, $f\left(x_{p}\right)=0$, and the dynamics take the form of (42), i.e.,

$$
\underbrace{\ddot{z}_{p}(k)}_{d(k)}=\hat{\rho}_{d}(k) \underbrace{T \operatorname{sat}\left(u_{T}(k)\right)}_{\phi(k)}
$$

Since the linearization of (55) results in four decoupled systems, $\hat{\rho}_{d}(k)$ can be structured as

$$
\hat{\rho}_{d}(k)=\operatorname{diag}\left(1 / \hat{m}(k), 1 / \hat{J}_{11}(k), 1 / \hat{J}_{22}(k), 1 / \hat{J}_{33}(k)\right)
$$

For implementation of the RLS, a value of $\lambda=0.98$ is used for the forgetting factor in (44a) 
and (44d). The identification algorithm operates at time steps $\Delta t=0.0001 \mathrm{~s}$. Additionally, the diagonal parameter estimate is initialized to $\hat{\rho}_{d}(0)$ where the value of the reciprocal of each diagonal element is given in Table 1.

\subsection{MRAC Design}

The MRAC design is identical for both $C_{\hat{K}, \Theta}$ and $C_{\hat{K}}$. It is important to note that while (24) is derived with the input matrix $B(\rho)$ from (6), the adaptation law is implemented discretely since estimates $\hat{\rho}$ are made available at fixed-time intervals $\Delta t$ from the system identification. After integrating $(24), \hat{K}(\rho)$ can be implemented discretely as

$$
\hat{K}^{\prime}(\hat{\rho}(k))=-\left(\frac{z}{z-1}\right) \Gamma_{K} x(k) \bar{e}^{\prime}(k) P_{z} B_{z}(\hat{\rho}(k))
$$

where $z /(z-1)$ represents the discrete equivalent of integration using the forward Euler method that results from the $\mathrm{z}$-transform of $1 / s$ from the Laplace domain. The vectors $x(k)$ and $\bar{e}(k)$ are sampled at the input of $\bar{K}(\rho) . P_{z}$ is the discrete equivalent of $P$ in (20) which must be solved using the discrete algebraic Lyapunov equation $A_{r z} P_{z} A_{r z}^{\prime}-P z+\mathscr{Q}_{K}=0$ where $A_{r z}$ is the dicrete version of $A_{r}$ from (8) which is determined by the $\mathrm{ZOH}$ method. The final term $B_{z}(\hat{\rho}(k))$ is from (28) with dependence on $\hat{\rho}$ rather than $\rho$.

The main aspect of the MRAC design is the choice of the reference model which specifies the ideal performance of the plant. Following from (12) and (8), the $A$ and $B$ matrices are taken from (6) when operating at the initial inertia parameter configuration $\rho_{o}$, thus $B=B\left(\rho_{o}\right)$. The remainder of the design is to calculate the ideal gain $K^{*}$, which is used for both determining the reference model dynamics and the LTI control signal $u_{L}$. The design of $K^{*}$ is performed using the LQR optimal control method [15], for which the weighting matrices are chosen as $Q_{l q r}=I_{12}, R_{l q r}=0.001 \times I_{4}$. This choice of $R_{l q r}$ and $Q_{l q r}$ places emphasis on quadcopter performance (small reference tracking error) rather than minimizing control effort. The resulting reference model and nominal closed-loop system have the 
following 12 closed-loop pole locations: $-27.48,-632.45$, a repeated pole at -1216.26 , and four repeated complex conjugate pairs of poles at $-0.87 \pm 0.50 i$.

Additional MRAC parameters must be selected including $\mathscr{Q}_{K}$ from (20) (and its discrete equivalent above) and the diagonal adaptation rate matrix $\Gamma_{K}$ in (24). The only requirement on $\mathscr{Q}_{K}$ is $\mathscr{Q}_{K}=\mathscr{Q}_{K}^{\prime}>0$. This parameter is arbitrarily chosen as the identity matrix, i.e., $\mathscr{Q}_{K}=I_{12}$. To tune $\Gamma_{K}$, it was initially set equal to the identity matrix $I_{12}$ and the simulation described in Section 5.3 was evaluated. While the plant states converged to the reference model trajectories within a reasonable period of time for quadcopter performance, significant overshoot and oscillation existed on all of the tracked states. In an effort to improve MRAC performance, a tuning experiment was carried out to determine a $\Gamma_{K}$ that reduces these undesirable overshoots and oscillations. To minimize the number of tuning parameters, the elements of $\Gamma_{K}$ were divided into three groups that correspond to the adaptation rates of the respective states in each group: 1 . the error states $x_{I}=\int\left(r-z_{p}\right) d t ; 2$. the position states $z_{p}=\left(x_{3}, \theta_{1}, \theta_{2}, \theta_{3}\right)$; and, 3 . the velocity states $\dot{z}_{p}=\left(\dot{x}_{3}, \dot{\theta}_{1}, \dot{\theta}_{2}, \dot{\theta}_{3}\right)$. Thus, instead of tuning twelve individual parameters (one per state), only three groups of parameters required tuning. The simulation described in Section 5.3 was evaluated with each of the groups' adaptation rates at all combinations of 1, 10, and 100. The diagonal adaptation rate matrix $\Gamma_{K}$ that resulted in the smallest overshoots and fastest return to reference model tracking for these simulations was determined as

$$
\Gamma_{K}=\operatorname{diag}(\underbrace{I_{4}}_{x_{I}}, I_{4} \otimes\left[\begin{array}{cc}
\underbrace{1}_{z_{p, i}} & 0 \\
0 & \underbrace{10}_{\dot{z}_{p, i}}
\end{array}\right])
$$

where the underbraces indicate the adaptation rate for each state group. At first glance, the resulting optimum $\Gamma_{K}$ is unexpected. Intuitively, one would expect the need for larger adaptation rates for $x_{I}$ in order to quickly adapt the error states and hence reduce overshoot and oscillations. However, a large adaption rate for $x_{I}$ resulted in large control effort, 
particularly in the transient portion of the simulation. This in turn caused long periods of saturation; and, since anti-windup compensation is absent in $C_{\hat{K}}$, the control system was unable to recover. Consequently, for the portion of $\Gamma_{K}$ responsible for the adaptation of $x_{I}$, the identity matrix $I_{4}$ resulted in no saturation during the transient portion of the simulation, and hence, best performance.

\subsection{PDAWC Design}

To implement the gain-scheduled $\Theta(\rho)$, first note that under the assumption that the payload is attached to the vehicle's center of mass, each mass moment of inertia $J_{i i}(t)$ changes proportional to $m(t)$ viz

$$
J_{i i, c}(t)=\left(\frac{\hat{m}}{m_{o}}\right) J_{i i, o}
$$

where $J_{i i, c}(t)$ are mass moments of inertia calculated from the estimated vehicle mass $\hat{m}$. Consequently, the parameter grid defined as $\mu_{\rho}$ used to generate the gain schedule denoted by $F_{G S}\left(\mu_{\rho}\right)$ can be reduced from a 4-dimensional matrix dependent on $\rho=\left[1 / m 1 / J_{11} 1 / J_{22} 1 / J_{33}\right]$ to a 1 -dimensional matrix dependent solely on $1 / m$. Similarly, the scheduling variable can be reduced from the vector $\hat{\rho}$ to the scalar $1 / \hat{m}$ where the mass moments of inertia are again calculated using (62). To reduce the possibility that any estimated values $\hat{m}$ do not fall outside the range of $\mu_{\rho}$ during simulation, a large buffer is included on both sides of the expected mass range of $m(t) \in[1.15,1.60]$. The mass grid $\mu_{\rho}$ is constructed using the range $\underline{m}=0.5 \mathrm{~kg}$ to $\bar{m}=5 \mathrm{~kg}$ with increments of $0.01 \mathrm{~kg}$. The LMI (41) is then solved offline for all discrete values of $1 / m$ on the grid $\mu_{\rho}$ to generate the 
gain schedule $F_{G S}\left(\mu_{\rho}\right)$, and the anti-windup compensator is implemented as

$$
\Theta(1 / \hat{m}) \sim \begin{cases}x_{a}(k+1) & =\left(A_{z}+B_{z}(1 / \hat{m}) F(1 / \hat{m})\right) x_{a}(k)+B_{z}(1 / \hat{m}) \tilde{u}_{F}(k) \\ u_{d}(k) & =F(1 / \hat{m}) x_{a}(k) \\ y_{d}(k) & =C_{z} x_{a}(k)\end{cases}
$$

where the anti-windup gain $F(1 / \hat{m})$ is obtained from the gain schedule $F_{G S}(1 / \hat{m})$.

The LMI (41) requires a narrowed sector to guarantee local stability for the marginally stable linearized quadcopter dynamics (58). For this simulation study, the local sector term $\beta_{i}$ defined in (39) is $\beta_{i}=100 \forall i=1, \ldots, m$. This ensures local stability so long as $u_{T, i}<100 \bar{u}_{T, i} \forall i=1, \ldots, m$.

To provide the control designer with an ability to tune anti-windup performance to the specific control system, [24] includes the choice of two weighting matrices (similar to the LQR method for the design of the reference model in MRAC). The performance weight $W_{p}$ prioritizes a fast return to nominal control performance, and the robustness weight $W_{r}$ emphasizes robustness to additive uncertainty. The choice of $W_{p}$ and $W_{r}$ can have a significant effect on anti-windup performance. Thus, an analysis of the influence of these weighting matrices on the performance of $C_{\hat{K}, \Theta}$ was conducted. The results of this analysis are presented in Table 2.

Table 2: Anti-windup design parameters' effect on performance $\left(W_{p}=I\right)$.

\begin{tabular}{c|ccccc}
\hline$W_{r}$ & 0.01 & 0.1 & 1 & 10 & 100 \\
Plot legend (Figs. 7 and 8) & & - & - & -- & \\
\hline$\gamma(1 / \bar{m})$ & 0.89 & 2.09 & 5.79 & 6.60 & 53.36 \\
$\gamma(1 / \underline{m})$ & 0.58 & 1.73 & 5.33 & 5.47 & 52.03 \\
\hline \hline
\end{tabular}

For each test, $W_{p}$ is held constant as the identity matrix, and $W_{r}$ is varied by orders of magnitude. Each set of $W_{r}$ results in an PDAWC with a corresponding $\gamma(1 / m)$ for both 
parameter extremes $\underline{m}$ and $\bar{m}$ as shown in Table 2 . It is clear from Table 2 that larger $W_{r}$ results in larger $\mathscr{L}_{2}$ gain of the anti-windup compensator. Because minimizing the $\mathscr{L}_{2}$ gain results in a smaller deviation from nominal control performance during saturation, anti-windup compensators designed with lower values of $W_{r}$ result in better anti-windup performance. To visualize the performance differences resulting from the choice of $W_{r}$ and the compensator's corresponding $\mathscr{L}_{2}$ gain, the simulation described in Section 5.3 is performed on the control system $C_{\hat{K}, \Theta}$ with $W_{r}=0.1,1$, and 10 .

\subsection{Simulation Results}

The $C_{\hat{K}, \Theta}$ and $C_{\hat{K}}$ controllers are tested with the same reference commands for the following quadcopter states: $r=\left[\begin{array}{llll}x_{3} & \theta_{1} & \theta_{2} & \theta_{3}\end{array}\right]^{\prime}$. The simulation begins with the quadcopter being released at $x_{3}=-1 \mathrm{~m}$ where it is commanded to hover for 5 seconds while the adaptive controller accounts for the transients due to gravity. At $t=5 \mathrm{~s}$, the quadcopter drops down to $x_{3}=0 \mathrm{~m}$ where it collects the payload shortly after at $t=10 \mathrm{~s}$. The quadcopter performs various test maneuvers for each of the tracked states and the simulation ends with the quadcopter landing at $r=\left[\begin{array}{llll}0 & 0 & 0 & 0\end{array}\right]^{\prime}$.

Because the input to the RLS algorithm is dependent on the control signal, both controller systems $C_{\hat{K}, \Theta}$ and $C_{\hat{K}}$ result in different estimates of the parameters. Rather than show the parameter estimates and the corresponding actual values, they are reciprocated and plotted in Fig. 6 to better visualize the mass increase after the payload is lifted. In Fig. 6 , discrepancies between the estimated and true parameter values exist at various instances during the simulation. As will be discussed shortly, when saturation occurs, the input to the RLS algorithm remains constant. However, the output of the RLS continues to change. Although the parameters are not actually changing when saturation occurs in this simulation, the algorithm has no knowledge of the saturation event, and thus, the parameter estimate continues to change to accommodate for the varying outputs.

Time histories of the tracked states $z_{p}$ and thrust signals sat $\left(u_{T}\right)$ for each $C_{\hat{K}, \Theta}$ are shown 

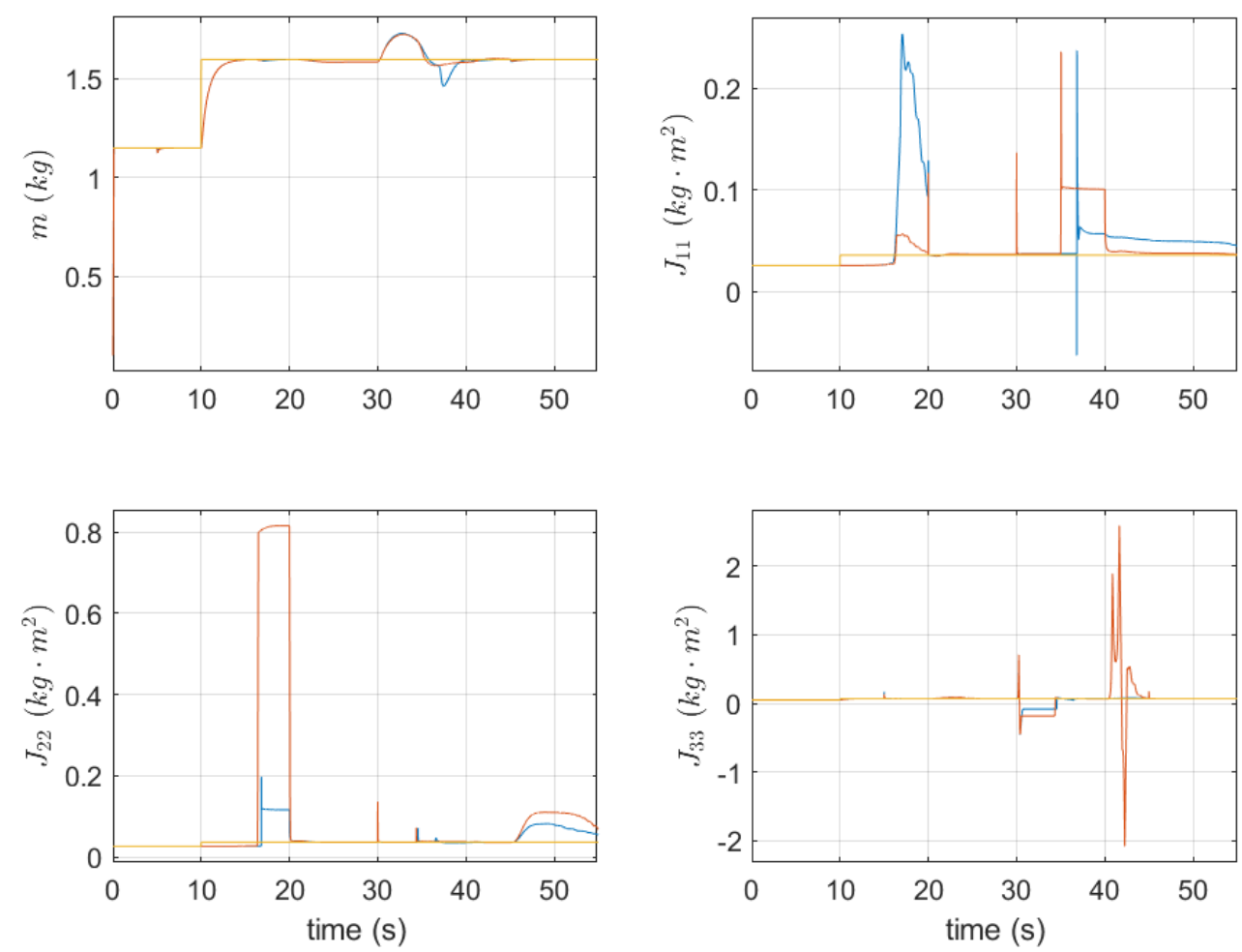

Figure 6: RLS parameter estimation. _ $1 / \hat{\rho}_{i} C_{\hat{K}} ; \_1 / \hat{\rho}_{i} C_{\hat{K}, \Theta} ;-1 / \rho_{i}$.

in Figs. 7 and 8, respectively. The legend for the different $C_{\hat{K}, \Theta}$ designs is included in Table 2. Additional signals included in the Fig. 7 are the reference signal $r$, the position state outputs of the reference model $z_{r}$ which correspond to the plant states defined in (57), and the output signals of the MRAC only controller (see figure caption for line-types for these signals). One additional signal is included in Fig. 8, which is the thrust signals of the $C_{\hat{K}}$ controller, labeled as $u_{T}^{C_{\hat{K}}}$.

When high emphasis is placed on PDAWC robustness $\left(W_{r}=10\right)$ the $\mathscr{L}_{2}$ gain of the compensator is relatively large. Hence, the response has a slow recovery time. In fact, after saturation has ended, this control system returns to reference tracking more slowly than each of the performance-to-robustness designs assessed in this analysis. On the other hand, placing PDAWC design emphasis on performance $\left(W_{r}=0.1\right)$ results in a relatively small $\mathscr{L}_{2}$ gain of the compensator, and, as expected, the response returns quickly to MRAC 

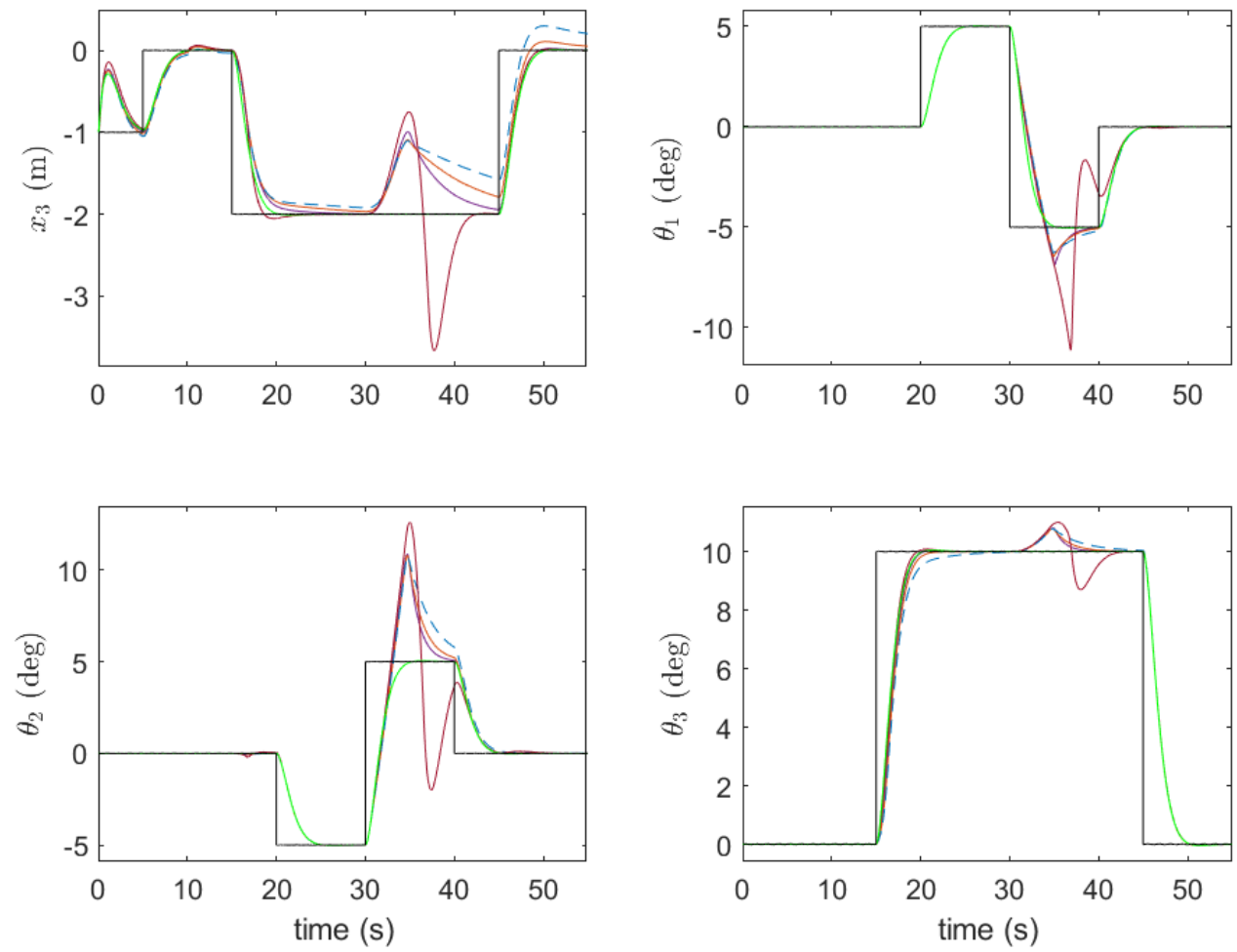

Figure 7: Effect of $W_{r}$ on system response. $\_r ; \_z_{r} ;-C_{\hat{K}}$; Additional legend elements in Table 2.

control performance. However, with high performance weighting, the system response during saturation results in overshoots around the $35 \mathrm{~s}$ mark for the $x_{3}$ and $\theta_{1}$ plots that are greater in magnitude that the overshoots of the PDAWC when $W_{r}=10$.

When analyzing the effect of $W_{r}$ on the thrust signals shown in Fig. 8, it is important to note that all variations of $W_{r}$ for the control system $C_{\hat{K}, \Theta}$ assessed in this study result in less saturation time than the $C_{\hat{K}}$ control system where AWC is absent. When comparing the saturated thrust signals in Fig. 8 with the results assessed in Fig. 7, the largest deviation from nominal control performance occurs simultaneously with the largest saturation event at $t=30 \mathrm{~s}$. When comparing the reference signals of the states $\theta_{1}$ and $\theta_{2}$ with the quadcopter model in Fig. 5, it can be determined that the first set of pulses for $\theta_{1}$ and $\theta_{2}$ between $t=20 \mathrm{~s}$ and $t=30 \mathrm{~s}$ result in forward flight, and the second set between $t=30 \mathrm{~s}$ and $t=40 \mathrm{~s}$ result in backwards flight. Therefore, at $t=30 \mathrm{~s}$, the quadcopter is commanded 

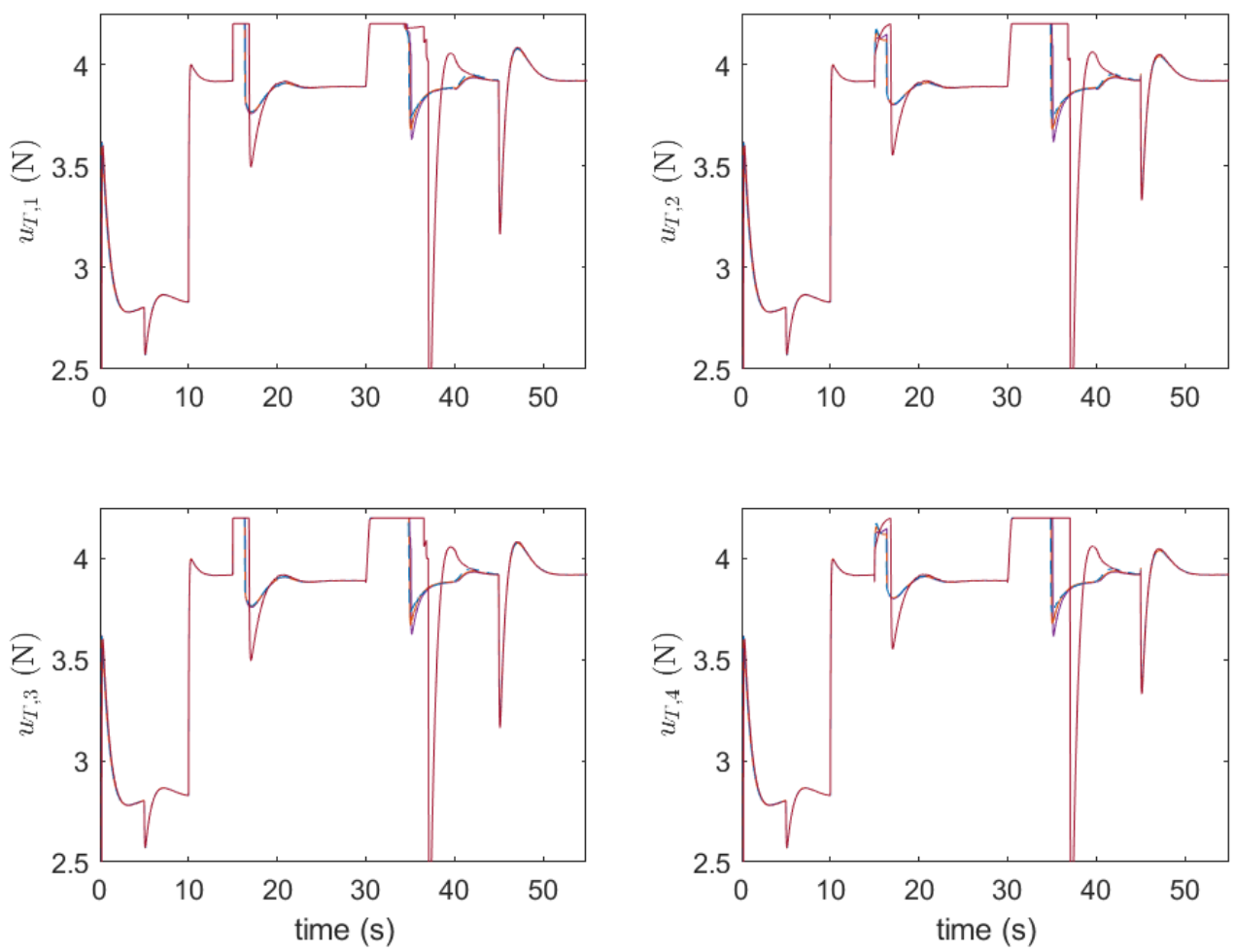

Figure 8: Effect of $W_{r}$ on thrust commands. $-u_{T}^{C_{\hat{K}}}$.

to abruptly change directions. With the additional weight of the package, this maneuver requires a large change in momentum that results in a long period of thrust saturation for each of the propellers. This saturation event causes both the deviation from nominal control performance shown in Fig. 7 and the deviation of the parameter estimates from the true parameter values shown in Fig. 6.

With the information presented in Figs. 7 and 8 and Table 2, the final design of the $C_{\hat{K}, \Theta}$ is chosen with $W_{r}=0.1$ which has relatively low $\gamma(1 / \underline{m})$ and $\gamma(1 / \bar{m})$. This design also returns to tracking the reference model trajectories $z_{r}$ more quickly than the other designs. 


\section{CONCLUSIONS}

A model reference adaptive controller and a parameter-dependent anti-windup compensator are presented in a control scheme for quadcopters with constrained inputs and time varying inertia. Using an anti-windup compensation architecture that allows for independent analysis of both the MRAC and anti-windup compensator, stability is ensured while $\mathscr{L}_{2}$ performance of the compensation is guaranteed. Stability is achieved by formulating an adaptive MRAC control law that ensures asymptotically stable error between the quadcopter and reference model outputs. Due to the time varying inertia parameters of the quadcopter, the resulting adaptive control law is dependent on the the varying parameters which are estimated by a recursive least squares system identification algorithm. To implement anti-windup for the time varying quadcopter, the estimated mass from the recursive least squares system identification algorithm are used to schedule the anti-windup compensator gain.

Simulations are carried out to evaluate the effectiveness of the control scheme for a quadcopter retrieving a package. Results show that the control scheme outperforms the control system with MRAC alone. This is further confirmed by investigating various performance-to-robustness weight ratios used in the anti-windup compensator design. While the case implemented assumes that the quadcopter's inertia properties are proportional to the the vehicle's mass, hence simplifying the gain scheduling of the anti-windup compensation, future work will require the need for gain scheduling that uses estimates of the inertia independent from the mass to enable the evaluation of a wider class of quadcopter applications. Likewise, the effects of the control scheme on energy efficiency will be investigated for quadcopter battery life since this is a real problem that must be addressed 
in order for successful use of the vehicles for future applications. 


\section{REFERENCES}

[1] Karl J. Astrom and Bjorn Wittenmark. Adaptive Control. Dover Publications, 2 edition, 2008.

[2] Peng Bo, Yang Lingyu, and Yang Xiaoke. Lpv-mrac method for aircraft with structural damage. In Chinese Control Conference, pages 3262-3267, Chengdu, China, 2016. Chinese Control Conference.

[3] Jiao Chen, Jiangyun Wang, and Weihong Wang. Model reference adaptive control for a class of aircraft with actuator saturation. In Chinese Control Conference, pages 2705-2710, Wuhan, China, 2018. Chinese Control Conference.

[4] Mohamad Dhaybi and Naseem Daher. Accurate real-time estimation of the inertia tensor of package delivery quadrotors. In American Control Conference, pages 1520_ 1525, Denver, CO, 2020. American Automatic Control Council.

[5] A.L. Do, J.M. Gomes da Silva Jr., O. Sename, and L. Dugard. Control design for lpv systems with input saturation and state constraints: an application to a semi-active suspension. In IEEE Conference on Decision and Control and European Control Conference, pages 3416-3421, Orlando, FL, 2011. IEEE.

[6] John Doyle, Bruce Francis, and Allen Tannenbaum. Feedback Control Theory. Macmillan Publishing Co., 1990.

[7] Kenneth Eure. Implementations of real-time system identification using recursive techniques. In American Control Conference, pages 4807-4812, Anchorage, AK, 2002. IEEE. 
[8] Gene F. Franklin, J. David Powell, and Abbas Emami-Naeini. Feedback Control of Dynamic Systems. Pearson, 8 edition, 2020.

[9] Jinchao Guo, Yanfeng Wang, Guangzhao Cui, and Xinhan Huang. An anti-windup design for linear parameter variation systems with input saturation. In International Conference on Bio-Inspired Computing: Theories and Applications, pages 281-284. IEEE, 2007.

[10] Guido Herrmann, Matthew C. Turner, and Ian Postlethwaite. Discrete-time and sampled-data anti-windup synthesis: stability and performance. International Journal of Systems Science, 37(2):91-113, 2006.

[11] Howard Kaufman, Itzhak Barkana, and Kenneth Sobel. Direct Adaptive Control Algorithms: Theory and Applications. Springer, 2 edition, 1997.

[12] Hassan Khalil. Nonlinear Systems. Prentice Hall, 3 edition, 2002.

[13] Eugene Lavretsky and Naira Hovakimyan. Positive $\mu$-modification for stable adaptation in dynamic inversion based adaptive control with input saturation. In American Control Conference, pages 3373-3378, Portland, OR, 2005. IEEE.

[14] Eugene Lavretsky and Naira Hovakimyan. Stable adaptation in the presence of input contraints. Systems \& Control Letters, 56:722-729, 2007.

[15] Eugene Lavretsky and Kevin A. Wise. Robust and Adaptive Control with Aerospace Applications. Springer, 2013.

[16] Vinícius F. Montagner, C. L. F. Oliveira, Pedro L. D. Peres, Sophie Tarbouriech, and Isabelle Queinnec. Gain-scheduled controllers for linear parameter-varying systems with saturating actuators: Lmi-based design. In American Control Conference, pages 6067-6072. IEEE, 2007. 
[17] Cesar C. Palerm and B. Wayne Bequette. Direct model reference adaptive control and saturation constraints. In 15th Triennial World Congress, Barcelona, Spain, 2002. Elsevier International Federation of Automatic Control (IFAC) Publications.

[18] Emmanuel Prempain, Matthew C. Turner, and Ian Postlethwaite. Coprime factor based anti-windup synthesis for parameter-dependent systems. Systems \& Control Letters, 58:810-817, 2009.

[19] Christopher M. Richards and Matthew C. Turner. Combined static and dynamic antiwindup compensation for quadcopters experiencing large disturbances. Journal of Guidance, Control, and Navigation, 43(4):673-684, 2020.

[20] Pouria Sarhadi, Abolfazl Ranjbar Noei, and Alireza Khosravi. Adaptive integral feedback controller for pitch and yaw channels of an auv with actuator saturations. ISA Transactions, 65:284-295, 2016.

[21] Jean Jacques E. Slotine and Weiping Li. Applied Nonlinear Control. Prentice Hall, 1991.

[22] B. L. Stevens and F. L. Lewis. Aircraft Control and Simulation. Wiley, 2003.

[23] Matthew C. Turner. Positive $\mu$-modification as an anti-windup mechanism. Systems \& Control Letters, 102:15-21, 2017.

[24] Matthew C. Turner, Guido Herrmann, and Ian Postlethwaite. Incorporating robustness requirements into antiwindup design. IEEE Transactions on Automatic Control, 52(10):1842-1855, 2007.

[25] Matthew C. Turner and Nkemdilim A. Ofodile. Decentralized approaches to antiwindup design with application to quadrotor unmanned aerial vehicles. IEEE Transactions on Control Systems Technology, 24(6):1980-1992, 2016. 
[26] Matthew C. Turner, Jorge Sofrony, and Emmanuel Prempain. Anti-windup for modelreference adaptive control schemes with rate-limits. Systems \& Control Letters, 137:1-9, 2020.

[27] Bernard Widrow and Samuel D. Stearns. Adaptive Signal Processing. Prentice-Hall, New Jersey, 1985.

[28] Fen Wu, Karolos M. Grigoriadis, and Andy Packard. Anti-windup controller design using linear parameter-varying control methods. International Journal of Control, 73(12):1104-1114, 2000.

[29] Fen Wu and Marco Soto. Extended anti-windup control schemes for lti and lft systems with actuator saturations. International Journal of Robust and Nonlinear Control, 14(15):1255-1281, 2009. 


\section{APPENDIX}

\section{A. EXPLORATION OF ADAPTIVE CONTROL METHODS}

Preliminary research focused heavily on the choice of the nominal adaptive controller to be applied in the control system developed in Section 3. Two general adaptive control architectures including adaptive model control (AMC) and model reference adaptive control (MRAC) were explored. Within the category of MRAC, many variations exist. However, three forms of MRAC including the MIT rule, the normalized MIT rule, and state feedback direct MRAC were explored. The performance of each of these adaptive controller types was evaluated with a simple simulation of single channel quadcopter dynamics with time-varying mass. The results of the adaptive controller simulations show the state feedback direct MRAC has both faster adaptation to mass changes and faster convergence to the commanded trajectories than the other types of adaptive control explored here. For this reason, the state feedback direct MRAC was chosen to be the nominal adaptive controller implemented in the main body of this paper.

\section{A.1 Adaptive Model Control}

Adaptive model control utilizes the least-mean-square (LMS) algorithm and an adaptive linear combiner to generate an adaptive model of the plant. The adaptive model is then inverted and placed before the plant. When the adaptive model is accurately tracking the plant, the product of the inverse adaptive model and the plant will simplify to the identity matrix and the reference command will feed directly through to the output. The adaptive model control structure is shown in Figure 9. 


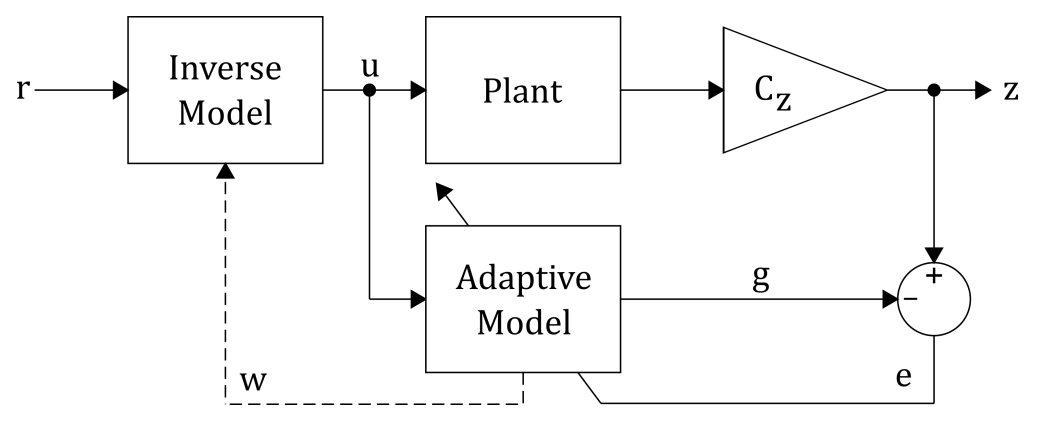

Figure 9: Adaptive Model Control

The adaptive controller consists of two parts - the adaptive model and the inverse model. The adaptive model can be described as a linear combiner

$$
\begin{aligned}
g_{k} & =\sum_{i=0}^{N} w_{i, k} u_{k-i} \\
& =\left[\begin{array}{llll}
u_{k} & u_{k-1} & \ldots & u_{k-N}
\end{array}\right]\left[\begin{array}{llll}
w_{0, k} & w_{1, k} & \ldots & w_{N, k}
\end{array}\right]^{\prime} \\
& =\mathbf{u}_{k}^{T} \mathbf{W}_{k}
\end{aligned}
$$

where $w_{i, k}$ is the $i^{t h}$ weight at the $k^{t h}$ time interval, $u_{k-i}$ is the control signal history, and the length of the weight vector $\mathbf{W}_{k}$ is called the tap-length $(\mathrm{L})$. The adaptive modeling error is defined as

$$
e_{k}=z_{k}-g_{k}
$$

Because the output $g_{k}$ is a linear combination of both the input signal $\mathbf{u}_{k}$ and the weight vector $\mathbf{W}_{k}$, the error can be redefined using (66)

$$
e_{k}=z_{k}-\mathbf{u}_{k}^{T} \mathbf{W}_{k}
$$

To minimize the adaptive modeling error, a steepest descent based algorithm is used. The gradient used to descend the performance surface is defined as

$$
\nabla_{k}=\frac{\partial e_{k}^{2}}{\partial \mathbf{W}_{k}}=2 e_{k} \frac{\partial e_{k}}{\partial \mathbf{W}_{k}}
$$


By taking the partial derivative of (68) with respect to $\mathbf{W}_{k}$, the gradient can be defined in terms of the error and input signals

$$
\nabla_{k}=-2 e_{k} \mathbf{u}_{k}
$$

The LMS algorithm is defined as

$$
\begin{aligned}
\mathbf{W}_{k+1} & =\mathbf{W}_{k}-\mu_{0} \nabla_{k} \\
& =\mathbf{W}_{k}+2 \mu_{0} e_{k} \mathbf{u}_{k} \\
& =\mathbf{W}_{k}+\mu e_{k} \mathbf{u}_{k} .
\end{aligned}
$$

Equation (71) demonstrates that the weight vector updates such that (67) decreases. For stability of the LMS algorithm, the rate of convergence $\mu$ must satisfy the relationship:

$$
0<\mu<\frac{1}{\left\|u_{k}\right\|^{2}}
$$

A more complete derivation of the LMS algorithm is found in [27].

The inverse model is developed by enforcing the desired condition that $r_{k}$ equals $g_{k}$. Equation (64) is then solved for the control signal $u_{k}$. This results in:

$$
u_{k}=\frac{1}{w_{0, k}}\left(r_{k}-\sum_{i=1}^{N} w_{i, k} u_{k-i}\right), w_{0, k} \neq 0 .
$$

For adaptive model control, the LMS algorithm would not converge for an open-loop marginally stable system. Full-state feedback was implemented to internally to ensure a bounded plant response. There are two parameters that affect system performance, the taplength $L$ and the convergence ratio $\mu$. The relationship between these two parameters and performance characteristics such as settling time and percent overshoot is not clear. Additionally, the two parameters have no theoretical method for calculating the ideal values. 
To ensure all dynamics can be modeled, $L$ must be greater than the order of the characteristic equation of the open loop plant. The constraint on $\mu$ (74) can only be checked if $u_{k}$ is known in advance. However, to ensure the constraint was not violated, $\mu$ was chosen by starting near zero and increasing the magnitude until the performance was satisfactory. After many iterations, parameters were chosen as

\begin{tabular}{cc} 
Parameter & Value \\
\hline \hline $\mathrm{L}$ & 3 \\
$\mu$ & $1 \mathrm{e}-4$
\end{tabular}

Table 3: AMC Parameters

The single channel quadcopter simulation of lifting a payload, regardless of the choice of $\mu$ and $L$, resulted in unbounded outputs. For this reason, AMC was discarded as an option for the nominal adaptive controller to be used in conjunction with the PDAWC designed in Section 3. Results, although underwhelming, generated for the simulation of the quadcopter with a constant mass are shown in Figure 10. 


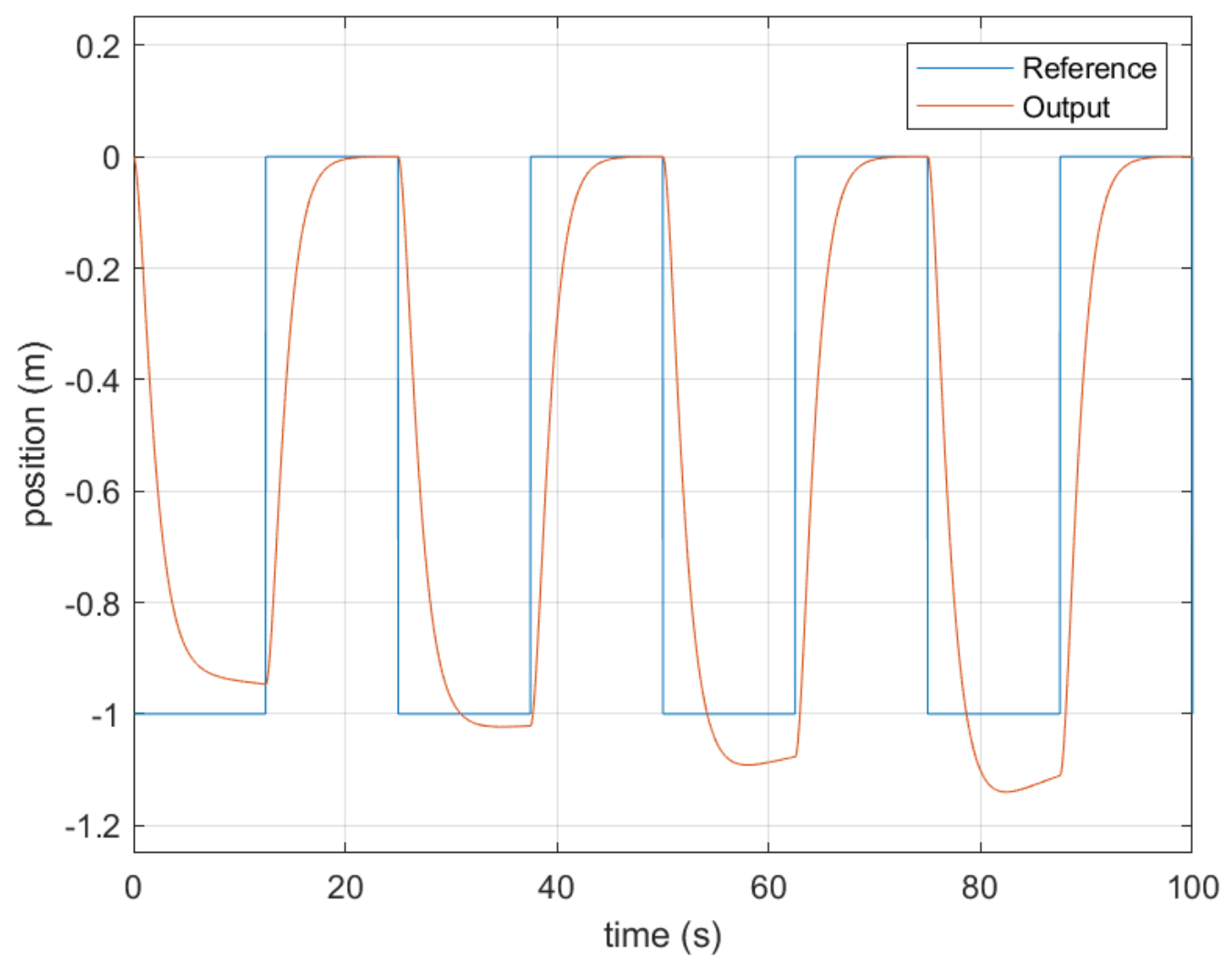

Figure 10: Adaptive Model Control for LTI Single Channel Quadcopter

Figure 10 shows the poor performance of the adaptive model control architecture. Attempting to improve the response time by increasing the convergence ratio $\mu$ caused worse performance or instability. The tuning of $\mu$ and $L$ was not a trivial task and many combinations result in unbounded responses. Literature for previous work on adaptive model control, specifically for open-loop marginally stable systems, was limited. Successful applications of adaptive model control was described in [27], but each of those systems had slowly time varying dynamics and very slow responses.

\section{A.2 Model Reference Adaptive Control}

The model-reference adaptive system is a system which contains the desired performance characteristics in the form a reference model. Parameters of the controller $(\theta)$ are adjusted based on the error which is defined as the difference between the output of the system ( $y$ ) 
and the output of the reference model $\left(y_{r}\right)$ [1]. Three methods for designing the adjustment mechanism in Figure 11 are presented below.

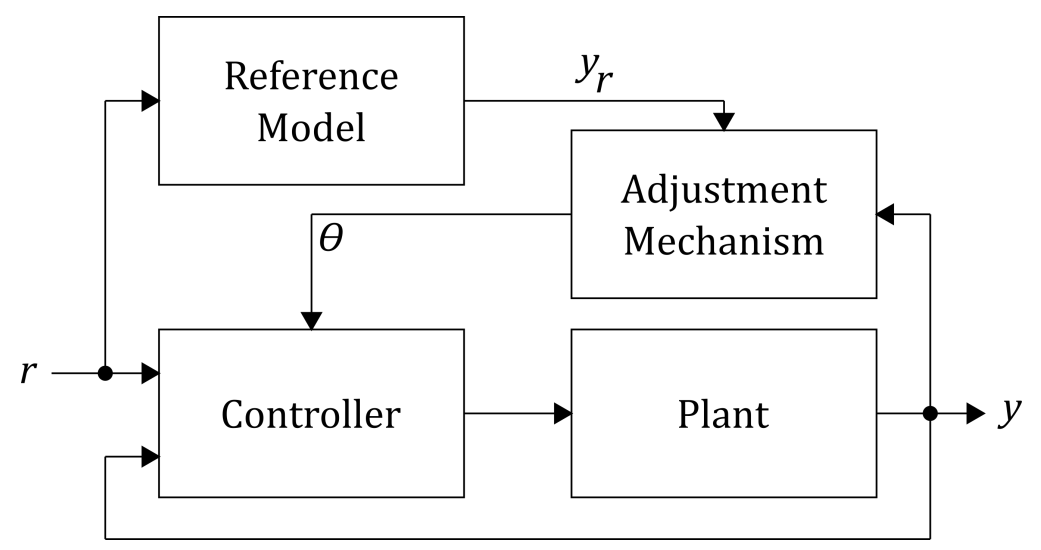

Figure 11: General MRAC Structure

\section{A.2.1 The MIT Rule}

The MIT rule, developed at MIT in 1958, was the first approach to MRAC. To present the MIT rule, consider Figure 11 which has one adjustable parameter $\theta$. This parameter must be chosen such that the output of the plant will converge to the desired closed-loop response specified by the reference model. Similar to minimizing the square error in (69), a cost function

$$
J(\theta)=\frac{1}{2} e^{2}
$$

will be minimized by updating $\theta$ in the direction of the negative gradient of $J$.

$$
\frac{d \theta}{d t}=-\gamma \frac{\partial J}{\partial \theta}=-\gamma e \frac{\partial e}{\partial \theta}
$$

where $\gamma$ is called the adaptation rate/gain and $\partial e / \partial \theta$ is called the sensitivity derivative. The error can be written in terms of the transfer functions from $r \rightarrow y$ and $r \rightarrow y_{r}$

$$
\begin{aligned}
e & =y-y_{r} \\
& =G_{p, c l} \theta r-G_{r} r
\end{aligned}
$$


where $G_{p, c l}$ is the closed loop transfer function of the plant, and $G_{r}$ is the transfer function of the reference model. The sensitivity derivative can be evaluated using (79)

$$
\frac{\partial e}{\partial \theta}=G_{p, c l} r
$$

Using the transfer function from $r \rightarrow y_{r}$ it is known that

$$
r=\frac{y_{r}}{G_{r}}
$$

If the reference model is designed so that its closed-loop plant dynamics are identitcal to those of the plant, when (81) is substituted into (80) the sensitivity derivative becomes

$$
\frac{\partial e}{\partial \theta}=y_{r}
$$

The adjustment mechanism is developed by substituting (82) and (78) into (77) and integrating which results in

$$
\theta=-\frac{\gamma}{s}\left(y-y_{r}\right)\left(y_{r}\right)
$$

To determine the adaptation gain $\gamma$ first rewrite (77) in terms of (79), (82) then rearrange the result to form the parameter equation:

$$
\frac{d \theta}{d t}+\gamma y_{r}\left(G_{p, c l} \theta r\right)=\gamma y_{r}^{2}
$$

If all signals are assumed to have reached steady state, (84) becomes

$$
\frac{d \theta}{d t}+\gamma y_{r, s s} r_{s s}\left(G_{p, c l} \theta\right)=\gamma y_{r, s s}^{2}
$$

which is simply a first order differential equation in terms of the parameter $\theta$. The Laplace transform yields the characteristic equation which governs the stability and performance of 
the parameter.

$$
s+\gamma y_{r, s s} r_{s s} G_{p, c l}=0
$$

A range of $\gamma$ that will guarantee stability of the adjustment mechanism can then be evaluated using the Routh array.

The transfer function for quadcopter $z$-axis translation is

$$
G_{p}(s)=\frac{1 / m}{s^{2}}
$$

A PD controller was used to achieve the performance criteria of $10 \%$ overshoot and to settle within $2 \%$ in 3 seconds.

$$
K_{p}(s)=5(s+1)
$$

The closed loop plant transfer function becomes

$$
G_{p, c l}(s)=\frac{\frac{5}{m}(s+1)}{s^{2}+\frac{5}{m} s+\frac{5}{m}}
$$

For perfect model following, the reference model must have the same dynamics as the closed-loop plant. To meet this condition, the reference model was chosen as

$$
G_{r}(s)=\frac{\frac{5}{m}(s+1)}{s^{2}+\frac{5}{m} s+\frac{5}{m}} .
$$

where $m=1.15$. When the plant mass equals the initial mass of $1.15 \mathrm{~kg}$, the plant output can perfectly track the reference model output otherwise there will be deviations. If average values of $y_{r, s s}$ and $r_{s s}$ are known, the adaptation rate $\gamma$ can be calculated using (86). For the simulation results presented in Figure 12, $y_{r, s s}$ and $r_{s s}$ are both equal to 1 . Then, substituting $G_{p, c l}$ into (86) and multiplying by the characteristic equation of $G_{p, c l}$, the parameter 
characteristic equation becomes

$$
s^{3}+\frac{5}{m} s^{2}+\frac{5}{m}(1+\gamma) s+\frac{5}{m} \gamma=0 .
$$

Using a Routh array [8], the range of stable values for $\gamma$ is

$$
0<\gamma<1-\frac{m}{5} .
$$

There is a clear trade off between adaptation rate and stability for different values of mass. As the mass of the system increases, the range of stable adaptation rates tightens. Prioritizing robustness over performance, the adaptation gain was chosen to be 0.1 . The single channel MIT Rule controlled quadcopter simulation results of lifting a payload of $3 \mathrm{Kg}$ at $t=50 s$ are shown in Figure 12.

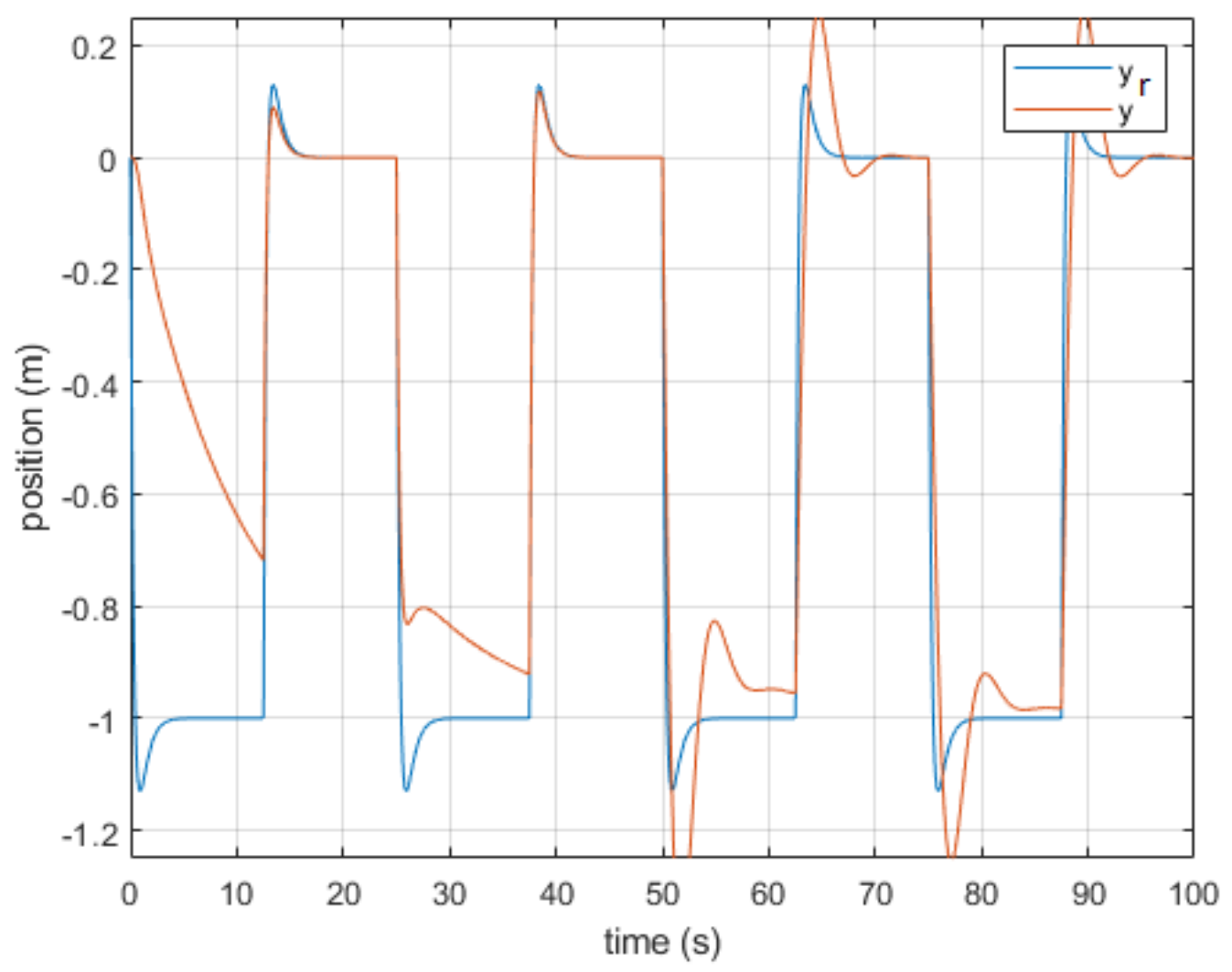

Figure 12: MIT Rule 
The MIT rule shows significant improvement when compared to the AMC results in Figure 10. However, when analyzing (86), the stability and adaptation speed of the system is dependent on the reference signal. This poses potential problems for large reference signals that will force smaller values of $\gamma$ to maintain stability and compromise adaptation rate.

\section{A.2.2 The Normalized MIT Rule}

Noting from (86), the reference signal has a direct effect on the stability and performance of the adjustment mechanism. The desired condition is $r=y=y_{r}$ at steady state. Thus, the square of the reference signal will affect the adjustment mechanism. If the reference signal is known to have large changes, the MIT rule will have performance limitations. To mitigate these effects, the following normalized MIT rule was developed

$$
\frac{d \theta}{d t}=\frac{\gamma \phi e}{\alpha+\phi^{T} \phi}
$$

where $\phi=-\partial e / \partial \theta$, and $\alpha>0$ is introduced to avoid undefined conditions when $\phi$ is small. Following the development of the parameter equation for the MIT rule in (84), (85), and (86), the characteristic equation for the normalized adjustment mechanism becomes

$$
s+\gamma \frac{y_{r, s s} r_{s s}}{\alpha+y_{r, s s}^{T} y_{r, s s} G_{p, c l}}=0 .
$$

Similarly, a range of $\gamma$ that will guarantee stability of the normalized adjustment mechanism can then be evaluated using the Routh array. Because $y_{r, s s}=r_{s s}$, the effect of the reference command on the adjustment mechanism stability and performance will be eliminated by the inclusion of $y_{m, s s}^{T} y_{m, s s}$ in the denominator.

When implementing the normalized MIT rule, (89) and (90) were used for the plant and reference models, respectively. The adaptation gain was chosen using the Routh array as before but using (94) instead of (86). The parameter characteristic equation is (91) and 
the range of stable values for gamma is (92). The adaptation gain was again chosen to be 0.1. The single channel normalized MIT Rule controlled quadcopter simulation results of lifting a payload of $3 \mathrm{Kg}$ at $t=50 \mathrm{~s}$ are shown in Figure 13.

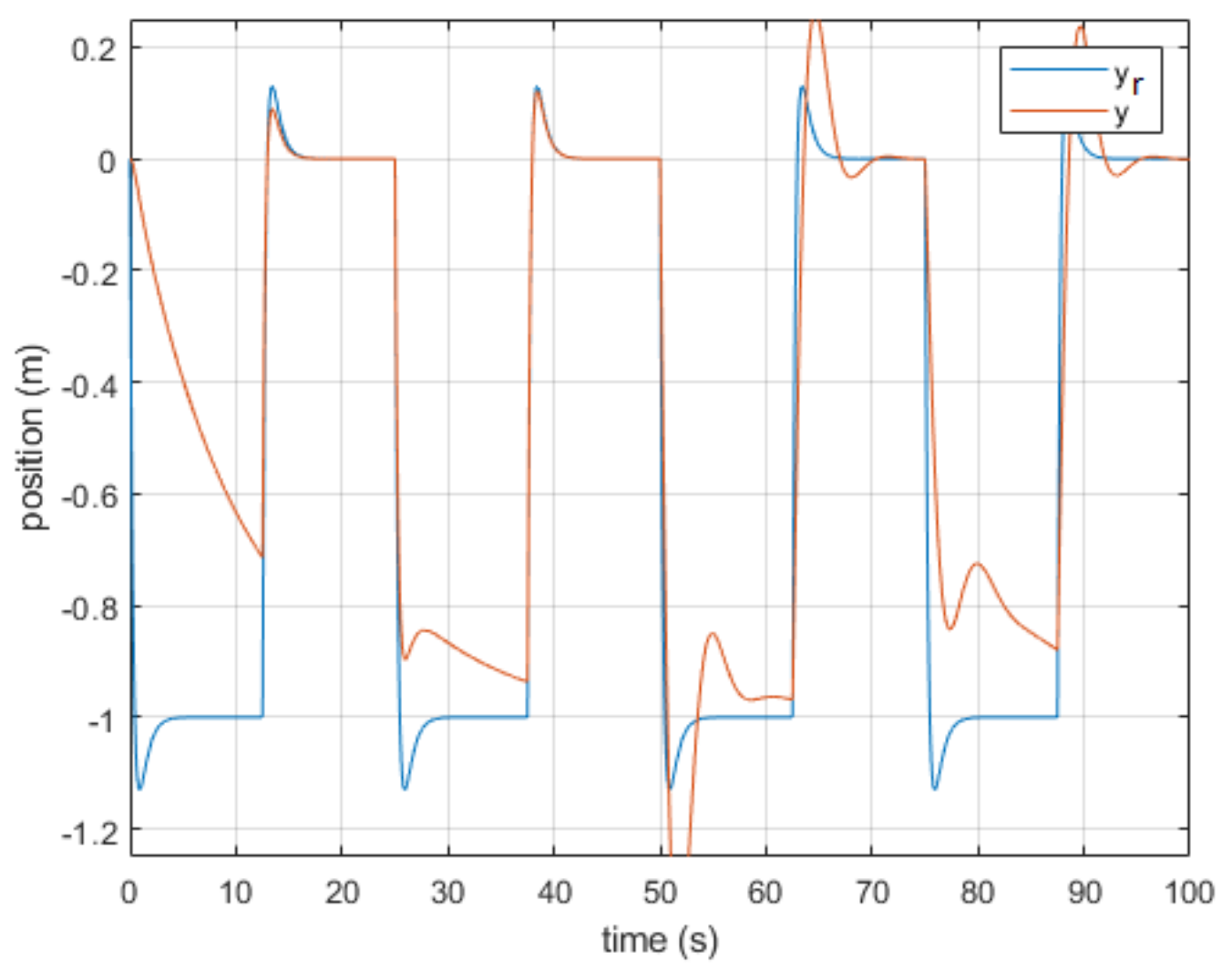

Figure 13: Normalized MIT Rule

The performance of the normalized MIT rule in Figure 13 is similar to the MIT rule performance in Figure 12. However, at the fourth pulse $(t=75 s)$, the plant output actually gets further from the reference model output. Although the normalized MIT rule performs worse than the MIT rule for this example, the elimination of dependence on the reference will allow the normalized MIT rule to have more consistent performance for a wider range of reference signals. 


\section{A.2.3 State Feedback Direct MRAC}

Because the state feedback direct MRAC is designed thoroughly in the body of the paper, theory is not presented here. For the full theoretical development and proof of stability, see Section 3.1. For comparison to the results of AMC, the MIT rule, and the normalized MIT rule, the same simulation is shown here in Figure 14. The reference model is designed as follows

$$
\begin{aligned}
& \dot{x}_{r}=\left[\begin{array}{cc}
0 & 1 \\
-\left(\omega_{n}\right)^{2} & -2 \zeta \omega_{n}
\end{array}\right] x_{r}+\left[\begin{array}{c}
0 \\
\omega_{n}{ }^{2}
\end{array}\right] r \\
& y_{r}=\left[\begin{array}{ll}
1 & 0
\end{array}\right] x_{r}+\left[\begin{array}{l}
0 \\
0
\end{array}\right] r
\end{aligned}
$$

where $m=1.15$ and $\zeta$ and $\omega_{n}$ were chosen to satisfy the following performance specifications:

\begin{tabular}{cc} 
Performance & Value \\
\hline \hline Overshoot & $10 \%$ \\
Settling time & $3.0 \mathrm{~s}$ \\
Settling amount & $2 \%$ \\
\hline \hline$\zeta$ & 0.59 \\
$\omega_{n}$ & 2.21
\end{tabular}

Table 4: State Feedback Direct MRAC Performance Specifications

\begin{tabular}{|c|c|}
\hline Parameter & Value \\
\hline$\Gamma_{x}$ & $\operatorname{diag}(100,100,100)$ \\
\hline
\end{tabular}

The following adaptation rates were chosen after a few iterations:

Table 5: State Feedback Direct MRAC Adaptation Gains

The single channel state feedback direct MRAC controlled quadcopter simulation results of lifting a payload of $3 \mathrm{Kg}$ at $t=50 \mathrm{~s}$ are shown in Figure 14. 


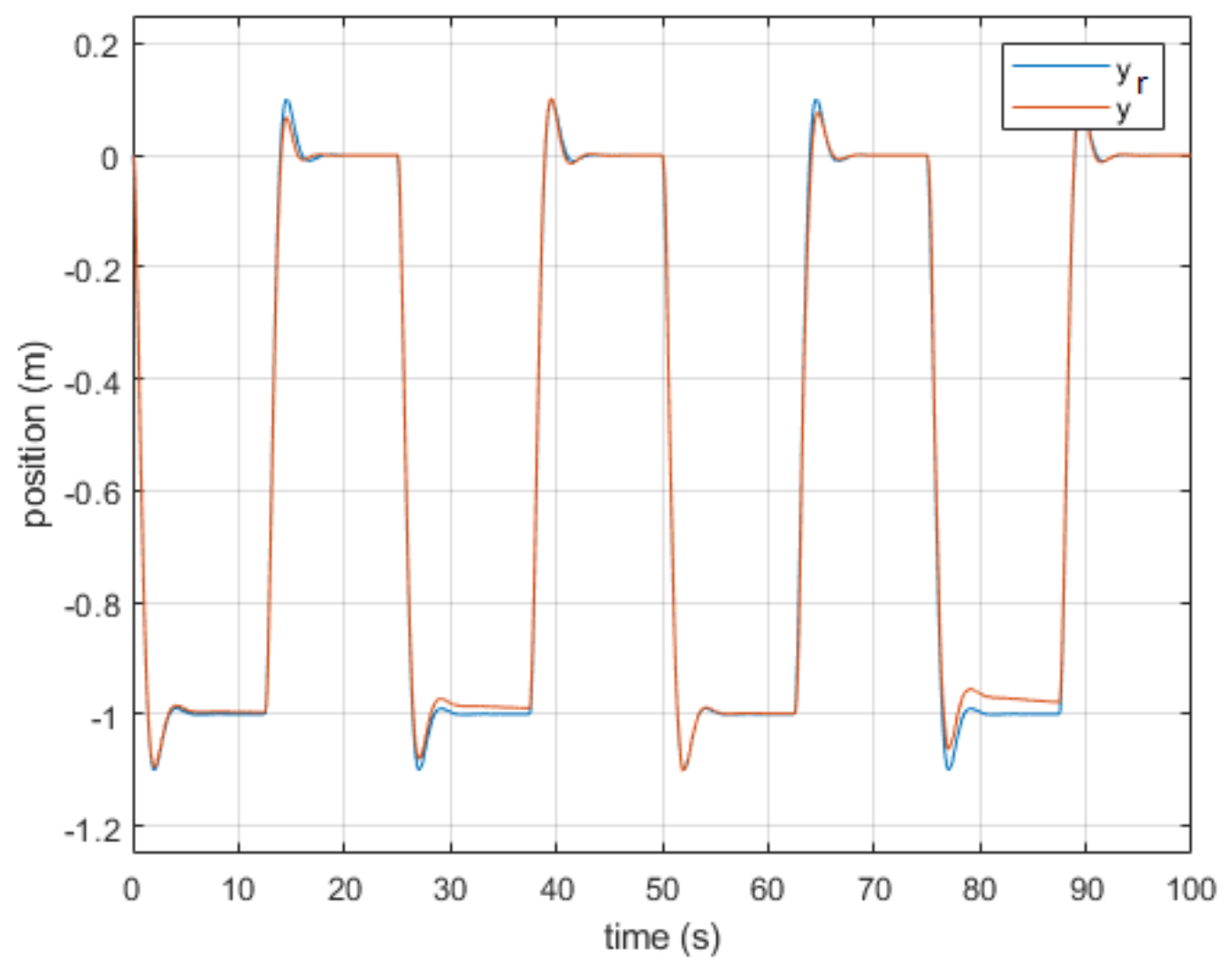

Figure 14: State Feedback Direct MRAC

Figure 14 demonstrates the ability of state feedback direct MRAC ensure the convergence of plant trajectories to the reference model trajectories. When the payload is added at $t=50 \mathrm{~s}$, this variation of MRAC has very small deviations from reference model tracking when compared to both the MIT rule and the normalzied MIT rule.

\section{A.3 Performance Comparison}

To provide a quantitative analysis of the performances of the MRAC variations (The LMS was not included due to instability during mass change simulation) the percent differences of the time integrals of the error signals and the time integrals of the desired signals are shown in Figure 15. 


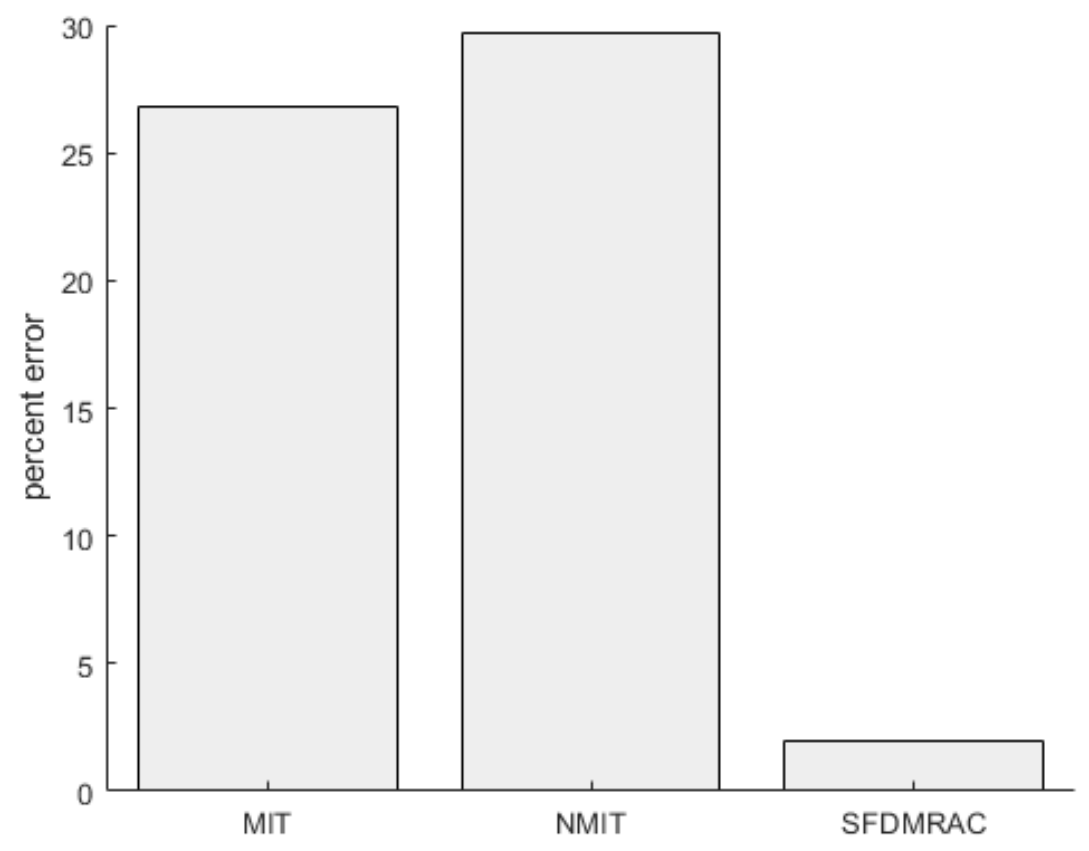

Figure 15: LTV Percent Difference of Error and Reference

Despite increasing the system mass significantly, the state feedback direct MRAC continually tracks the reference model through the duration of the simulation with only small deviations after the addition of cargo. For this reason, this variation of MRAC is chosen as the nominal adaptive controller to be implemented in Section 3. 


\section{CURRICULUM VITA}

NAME: $\quad$ Benjamin Edwards Farber

ADDRESS: $\quad 12504$ Mayo Court

Prospect, KY 40059

DOB: $\quad$ Louisville, Kentucky - June 20, 1997

EDUCATION: B.S. Mechanical Engineering

University of Louisville

J.B. Speed School of Engineering

2015-2019

M.S. Mechanical Engineering

University of Louisville

J.B. Speed School of Engineering

2019-present

EXPERIENCE: Graduate Teaching Assistant

University of Louisville

J.B. Speed School of Engineering

2020

Mechanical Engineering Intern

CMTA Consulting Engineering

2017-2018

AWARDS: $\quad$ Hsing Chuang Award for Excellence in Graduate Study 2020 